\title{
Quantum Monte Carlo calculations of electromagnetic moments and transitions in $A \leq 9$ nuclei with meson-exchange currents derived from chiral effective field theory
}

\author{
S. Pastore 1 因 Steven C. Pieper ${ }^{1}$ 团 R. Schiavilla ${ }^{2,3}$ and R. B. Wiringa粵 \\ ${ }^{1}$ Physics Division, Argonne National Laboratory, Argonne, Illinois 60439 \\ ${ }^{2}$ Theory Center, Jefferson Laboratory, Newport News, Virginia 23606 \\ ${ }^{3}$ Department of Physics, Old Dominion University, Norfolk, Virginia 23529
}

(Dated: November 9, 2018)

\begin{abstract}
Quantum Monte Carlo calculations of electromagnetic moments and transitions are reported for $A \leq 9$ nuclei. The realistic Argonne $v_{18}$ two-nucleon and Illinois-7 three-nucleon potentials are used to generate the nuclear wave functions. Contributions of two-body meson-exchange current (MEC) operators are included for magnetic moments and $M 1$ transitions. The MEC operators have been derived in both a standard nuclear physics approach and a chiral effective field theory formulation with pions and nucleons including up to one-loop corrections. The two-body MEC contributions provide significant corrections and lead to very good agreement with experiment. Their effect is particularly pronounced in the $A=9, T=3 / 2$ systems, in which they provide up to $\sim 20 \%(\sim 40 \%)$ of the total predicted value for the ${ }^{9} \mathrm{Li}\left({ }^{9} \mathrm{C}\right)$ magnetic moment.

PACS numbers: 21.10.Ky, 23.20.-g, 23.20.Js, 27.20.+n
\end{abstract}

\section{INTRODUCTION}

Quantum Monte Carlo (QMC) calculations of electroweak transitions in $A=6,7$ nuclei were reported in Ref. 1] and corrections for the magnetic moments (m.m.'s) and $M 1$ transitions from two-body mesonexchange current (MEC) operators were given in Ref. [2]. The QMC method is a two-step process, with an initial variational Monte Carlo (VMC) calculation to find a good trial function, followed by a Green's function Monte Carlo (GFMC) calculation to refine the solution. When used with the Argonne $v_{18}$ two-nucleon [3] and Illinois-2 three-nucleon [4] potentials, the final GFMC results reproduce the ground- and excited-state energies for $A \leq 10$ nuclei very well [5] 8 ].

In the present paper, we extend these calculations to $A=8,9$ nuclei using the improved Illinois- 7 threenucleon potential [9]. The electromagnetic (EM) current operator includes, in addition to the standard onebody convection and spin-magnetization terms for individual protons and neutrons, a two-body MEC component. The latter is constructed within two distinct frameworks, namely the same standard nuclear physics approach (SNPA) illustrated in Refs. 2, 10], and the chiral effective field theory $(\chi \mathrm{EFT})$ formulation of Refs. 1113.

We report energies, radii, magnetic and quadrupole moments, and a number of $M 1$ and $E 2$ transitions. The MEC contributions can make significant corrections to the m.m.'s and $M 1$ transitions, and we find general agree-

\footnotetext{
*Electronic address: pastore@anl.gov

† Electronic address: spieper@anl.gov

‡Electronic address: schiavil@jlab.org

$\S$ Electronic address: wiringa@anl.gov
}

ment between the two formulations and with experiment. However the $\chi \mathrm{EFT}$ formulation provides better agreement for the calculated m.m.'s, for which both MEC models are tested. The M1 transitions are calculated only with the $\chi$ EFT MEC operators, showing improved agreement with experiment in all cases.

A brief review of the QMC calculational method is given in Sec. III The EM current operator is discussed in Sec. III. Results and conclusions are given in Secs. IV and $\mathrm{V}$

\section{QUANTUM MONTE CARLO METHOD}

We seek accurate solutions of the many-nucleon Schrödinger equation

$$
H \Psi\left(J^{\pi} ; T, T_{z}\right)=E \Psi\left(J^{\pi} ; T, T_{z}\right),
$$

where $\Psi\left(J^{\pi} ; T, T_{z}\right)$ is a nuclear wave function with specific spin-parity $J^{\pi}$, isospin $T$, and charge state $T_{z}$. The Hamiltonian used here has the form

$$
H=\sum_{i} K_{i}+\sum_{i<j} v_{i j}+\sum_{i<j<k} V_{i j k},
$$

where $K_{i}$ is the non-relativistic kinetic energy and $v_{i j}$ and $V_{i j k}$ are respectively the Argonne $v_{18}$ (AV18) [3] and Illinois-7 (IL7) 9] potentials.

The VMC trial function $\Psi_{V}\left(J^{\pi} ; T, T_{z}\right)$ for a given nucleus is constructed from products of two- and three-body correlation operators acting on an antisymmetric singleparticle state of the appropriate quantum numbers. The correlation operators are designed to reflect the influence of the interactions at short distances, while appropriate boundary conditions are imposed at long range [14, 15]. The $\Psi_{V}\left(J^{\pi} ; T, T_{z}\right)$ has embedded variational parameters 
that are adjusted to minimize the expectation value

$$
E_{V}=\frac{\left\langle\Psi_{V}|H| \Psi_{V}\right\rangle}{\left\langle\Psi_{V} \mid \Psi_{V}\right\rangle} \geq E_{0},
$$

which is evaluated by Metropolis Monte Carlo integration [16]. Here $E_{0}$ is the exact lowest eigenvalue of $H$ for the specified quantum numbers. A good variational trial function can be constructed with

$$
\left|\Psi_{V}\right\rangle=\mathcal{S} \prod_{i<j}^{A}\left[1+U_{i j}+\sum_{k \neq i, j}^{A} \tilde{U}_{i j k}^{T N I}\right]\left|\Psi_{J}\right\rangle,
$$

where the $\mathcal{S}$ is a symmetrization operator. The Jastrow wave function $\Psi_{J}$ is fully antisymmetric and has the $\left(J^{\pi} ; T, T_{z}\right)$ quantum numbers of the state of interest, while $U_{i j}$ and $\tilde{U}_{i j k}^{T N I}$ are the two- and threebody correlation operators. Although we construct the $\Psi_{V}\left(J^{\pi} ; T, T_{z}\right)$ to be an eigenstate of the isospin $T$, we allow isobaric analog states with different $T_{z}$ to have different wave functions, reflecting primarily the difference in Coulomb contributions, but also additional chargesymmetry-breaking parts of the AV18 interaction.

The GFMC method [17, 18] improves on the VMC wave functions by acting on $\Psi_{V}$ with the operator $\exp \left[-\left(H-E_{0}\right) \tau\right]$. In practice, a simplified version $H^{\prime}$ of the Hamiltonian $H$ is used in the operator, which includes the isoscalar part of the kinetic energy, a chargeindependent eight-operator projection of AV18 called $\mathrm{AV}^{\prime}$, a strength-adjusted version of the three-nucleon potential IL7' (adjusted so that $\left\langle H^{\prime}\right\rangle \sim\langle H\rangle$ ), and an isoscalar Coulomb term that integrates to the total charge of the given nucleus. More detail can be found in Refs. [15, 19].

The operator is applied in small slices of imaginary time $\tau$ to produce a propagated wave function:

$$
\Psi(\tau)=e^{-\left(H^{\prime}-E_{0}\right) \tau} \Psi_{V}=\left[e^{-\left(H^{\prime}-E_{0}\right) \triangle \tau}\right]^{n} \Psi_{V} .
$$

Obviously $\Psi(\tau=0)=\Psi_{V}$ and $\Psi(\tau \rightarrow \infty)=\Psi_{0}$. The algorithm for propagation produces samples of the wave function $\Psi(\tau)$ but does not provide gradient information. Therefore, quantities of interest are evaluated in terms of a "mixed" expectation value between $\Psi_{V}$ and $\Psi(\tau)$ :

$$
\langle O(\tau)\rangle_{M}=\frac{\left\langle\Psi(\tau)|O| \Psi_{V}\right\rangle}{\left\langle\Psi(\tau) \mid \Psi_{V}\right\rangle}
$$

where the operator $O$ acts on the trial function $\Psi_{V}$. The desired expectation values would, of course, have $\Psi(\tau)$ on both sides; by writing $\Psi(\tau)=\Psi_{V}+\delta \Psi(\tau)$ and neglecting terms of order $[\delta \Psi(\tau)]^{2}$, we obtain the approximate expression

$$
\begin{aligned}
\langle O(\tau)\rangle & =\frac{\langle\Psi(\tau)|O| \Psi(\tau)\rangle}{\langle\Psi(\tau) \mid \Psi(\tau)\rangle} \\
& \approx\langle O(\tau)\rangle_{M}+\left[\langle O(\tau)\rangle_{M}-\langle O\rangle_{V}\right],
\end{aligned}
$$

where $\langle O\rangle_{\mathrm{V}}$ is the variational expectation value.
For the energy, the mixed estimate of Eq. (6) with $O=$ $H^{\prime}$ is itself a strict upper bound to the ground state for the simpler Hamiltonian, as can be seen by commuting half the imaginary time operator from the left to right hand side, giving

$$
\left\langle H^{\prime}(\tau)\right\rangle_{M}=\frac{\left\langle\Psi(\tau / 2)\left|H^{\prime}\right| \Psi(\tau / 2)\right\rangle}{\langle\Psi(\tau / 2) \mid \Psi(\tau / 2)\rangle} .
$$

The total energy is then given by this mixed estimate for $H^{\prime}$ plus the small difference $\left\langle\left(H-H^{\prime}\right)\right\rangle$ evaluated by Eq.(7).

For off-diagonal matrix elements required by transitions the generalized mixed estimate is given by the expression

$$
\begin{gathered}
\frac{\left\langle\Psi^{f}(\tau)|O| \Psi^{i}(\tau)\right\rangle}{\sqrt{\left\langle\Psi^{f}(\tau) \mid \Psi^{f}(\tau)\right\rangle} \sqrt{\left\langle\Psi^{i}(\tau) \mid \Psi^{i}(\tau)\right\rangle}} \\
\approx\langle O(\tau)\rangle_{M_{i}}+\langle O(\tau)\rangle_{M_{f}}-\langle O\rangle_{V},
\end{gathered}
$$

where

$$
\langle O(\tau)\rangle_{M_{f}}=\frac{\left\langle\Psi^{f}(\tau)|O| \Psi_{V}^{i}\right\rangle}{\left\langle\Psi^{f}(\tau) \mid \Psi_{V}^{f}\right\rangle} \sqrt{\frac{\left\langle\Psi_{V}^{f} \mid \Psi_{V}^{f}\right\rangle}{\left\langle\Psi_{V}^{i} \mid \Psi_{V}^{i}\right\rangle}}
$$

and $\langle O(\tau)\rangle_{M_{i}}$ is defined similarly. For more details see Eqs. (19-24) and the accompanying discussions in Ref. [1].

Sources of systematic error in the GFMC evaluation of operator expectation values (other than $H^{\prime}$ ) include the use of mixed estimates and the constrained path algorithm for controlling the Fermion sign problem in the propagation of $\Psi(\tau)$. These are discussed in Ref. [19]; the convergence of the current calculations is addressed at the beginning of Sec. IV

\section{THE ELECTROMAGNETIC CURRENT OPERATOR}

The nuclear EM current operator is expressed as an expansion in many-body terms. The current utilized in this work includes up to two-body terms. In what follows, we use the notation

$$
\begin{array}{ll}
\mathbf{k}_{i}=\mathbf{p}_{i}^{\prime}-\mathbf{p}_{i}, & \mathbf{K}_{i}=\left(\mathbf{p}_{i}^{\prime}+\mathbf{p}_{i}\right) / 2, \\
\mathbf{k}=\left(\mathbf{k}_{1}-\mathbf{k}_{2}\right) / 2, & \mathbf{K}=\mathbf{K}_{1}+\mathbf{K}_{2},
\end{array}
$$

where $\mathbf{p}_{i}\left(\mathbf{p}_{i}^{\prime}\right)$ is the initial (final) momentum of nucleon $i$, and $\mathbf{q}=\mathbf{k}_{1}+\mathbf{k}_{2}$ is the momentum associated with the external EM field.

The one-body operator at leading order-or impulse approximation (IA) operator - consists of the convection and the spin-magnetization currents associated with an individual nucleon. It is derived from the non-relativistic reduction of the covariant single-nucleon current by expanding it in powers of $\mathbf{p}_{i} / m_{N}$, where $m_{N}$ is the nucleon 


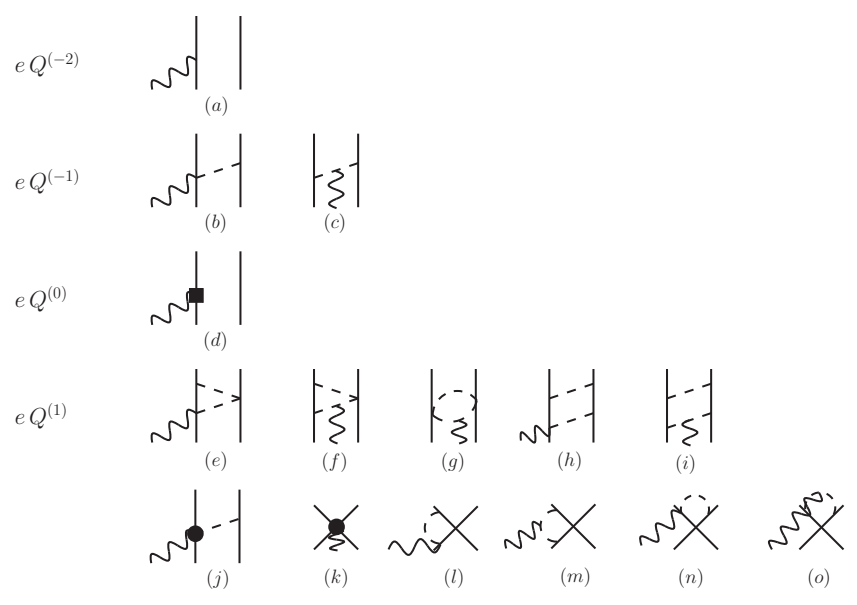

FIG. 1: Diagrams illustrating one- and two-body $\chi$ EFT EM currents entering at LO $\left(e Q^{-2}\right), \operatorname{NLO}\left(e Q^{-1}\right), \operatorname{N} 2 \operatorname{LO}\left(e Q^{0}\right)$, and N3LO $\left(e Q^{1}\right)$. Nucleons, pions, and photons are denoted by solid, dashed, and wavy lines, respectively.

mass and retaining the leading-order term. It reads

$$
\mathbf{j}^{\mathrm{IA}}=\frac{e}{2 m_{N}}\left[2 e_{N, 1} \mathbf{K}_{1}+i \mu_{N, 1} \boldsymbol{\sigma}_{1} \times \mathbf{q}\right],
$$

where

$e_{N}=\left(1+\tau_{z}\right) / 2, \quad \kappa_{N}=\left(\kappa_{S}+\kappa_{V} \tau_{z}\right) / 2, \mu_{N}=e_{N}+\kappa_{N}$.

Here $\kappa_{S}=-0.12$ n.m. and $\kappa_{V}=3.706$ n.m. are the isoscalar (IS) and isovector (IV) combinations of the anomalous m.m.'s of the proton and neutron, $e$ is the electric charge, and $\tau_{z}$ is the Pauli isospin projection equal to +1 for protons and -1 for neutrons.

The calculations of the m.m.'s of the $A \leq 9$ nuclei have been carried out utilizing two models for the two-body EM current operator, which are discussed in the next two subsections.

\section{A. $\chi$ EFT current operator}

Two-body EM currents have been derived in recent years within pionful chiral effective field formulations 11 13, 20 22 (for a comparison of the different formalisms we refer to the last four cited references). Here, we utilize the operators constructed within the formalism developed in Refs. [1, 12]. The $\chi$ EFT operators are expanded in powers of pions' and nucleons' momenta, and consist of long- and intermediate-range components which are described in terms of one- and two-pion exchange contributions, as well as contact currents encoding short-range mechanisms unresolved at the given order. These operators involve a number of low energy constants (LECs) which are fixed to the experimental data. The operators and fitting procedure have been recently described in Ref. [13]. We refer to that work for a complete listing of the operators utilized in the present
TABLE I: Dimensionless values of the isoscalar and isovector LECs entering the $\chi \mathrm{EFT}$ current operator at N3LO corresponding to cutoff $\Lambda=600 \mathrm{MeV}$.

\begin{tabular}{c|c|c|c|c|}
$\Lambda$ & $C_{15}^{\prime} \times \Lambda^{4}$ & $d_{9}^{\prime} \times \Lambda^{2}$ & $C_{16}^{\prime} \times \Lambda^{4}$ & $d_{8}^{\prime} \times \Lambda^{2}$ \\
\hline 600 & 5.238 & -0.2033 & -1.025 & 4.980 \\
\hline
\end{tabular}

calculations, and limit ourselves to discussing the various contributions and to summarizing the fitting strategy adopted to constrain the LECs.

The $\chi$ EFT EM operators are diagrammatically represented in Fig. 1. They are expressed as an expansion in the low-momentum scale $Q$. Referring to Fig. 1 the leading-order (LO) term is counted as $e Q^{-2}$, and corresponds to the IA one-body operator given in Eq. (14). The NLO term (of order $e Q^{-1}$ ) consists of the seagull and pion-in-flight one-pion-exchange (OPE) currents. These purely isovector currents involve two known LECs, the axial coupling constant $g_{A}=1.29$, and the pion decay amplitude $F_{\pi}=184.6 \mathrm{MeV}$. The value for $g_{A}$ is determined from the Goldberger-Treiman relation $g_{A}=$ $g_{\pi N N} F_{\pi} /\left(2 m_{N}\right)$, where the $\pi N N$ coupling constant is taken to have the value $g_{\pi N N}^{2} /(4 \pi)=13.63 \pm 0.20[23,24]$. The N2LO one-body contribution (of order $e Q^{0}$ ) is a relativistic correction to the IA operator, and is thus expressed in terms of the nucleons' experimental m.m.'s.

At N3LO $(e Q)$ we distinguish among four kinds of currents. The first one ("LOOP" in the tables), accounts for the one-loop contributions of diagrams (e)-(i) and (l)-(o). These terms lead to a purely isovector current which involves the known LECs $g_{A}$ and $F_{\pi}$. For diagram (m) we use the expression given in Ref. [13] which differs from that given in previous works [11, 12] by some of the present authors.

Next we account for the contact currents illustrated in panel $(\mathrm{k})$. We distinguish between minimal (MIN) and non-minimal (NM) currents. The former is linked to the $\chi$ EFT contact potential at N2LO via current conservation; therefore it involves the same LECs entering the $N N$ potential, and is [12, 13]

$$
\begin{aligned}
\mathbf{j}_{\mathrm{MIN}}^{\mathrm{N} 3 \mathrm{LO}}= & \frac{i e}{16}\left(\boldsymbol{\tau}_{1} \times \boldsymbol{\tau}_{2}\right)_{z}\left[\left(\mathbf{k}_{1}-\mathbf{k}_{2}\right)\right. \\
& \times\left[C_{2}+3 C_{4}+C_{7}+\left(C_{2}-C_{4}-C_{7}\right) \boldsymbol{\sigma}_{1} \cdot \boldsymbol{\sigma}_{2}\right] \\
& \left.+C_{7}\left[\boldsymbol{\sigma}_{1} \cdot\left(\mathbf{k}_{1}-\mathbf{k}_{2}\right) \boldsymbol{\sigma}_{2}+\boldsymbol{\sigma}_{2} \cdot\left(\mathbf{k}_{1}-\mathbf{k}_{2}\right) \boldsymbol{\sigma}_{1}\right]\right] \\
& -\frac{i C_{5}}{4}\left(\boldsymbol{\sigma}_{1}+\boldsymbol{\sigma}_{2}\right) \times\left(e_{1} \mathbf{k}_{1}+e_{2} \mathbf{k}_{2}\right),
\end{aligned}
$$

where the low-energy constants $C_{1}, \ldots, C_{7}$, have been constrained by fitting $n p$ and $p p$ elastic scattering data and the deuteron binding energy. We take their values from the Machleidt and Entem 2011 review paper [25]. Unknown EM LECs enter the NM current at N3LO which is given by

$\mathbf{j}_{\mathrm{NM}}^{\mathrm{N} 3 \mathrm{LO}}=-i e\left[C_{15}^{\prime} \boldsymbol{\sigma}_{1}+C_{16}^{\prime}\left(\tau_{1, z}-\tau_{2, z}\right) \boldsymbol{\sigma}_{1}\right] \times \mathbf{q}+1 \rightleftharpoons 2$, 
and the determination of the LECs $C_{15}^{\prime}$ and $C_{16}^{\prime}$ is discussed below.

The N3LO OPE current, diagram (j) in Fig. 1 is given by $[12,13]$

$$
\begin{aligned}
\mathbf{j}_{\mathrm{OPE}}^{\mathrm{N} 3 \mathrm{LO}}= & i e \frac{g_{A}}{F_{\pi}^{2}} \frac{\boldsymbol{\sigma}_{2} \cdot \mathbf{k}_{2}}{\omega_{k_{2}}^{2}}\left[\left(d_{8}^{\prime} \tau_{2, z}+d_{9}^{\prime} \boldsymbol{\tau}_{1} \cdot \boldsymbol{\tau}_{2}\right) \mathbf{k}_{2}\right. \\
& \left.-d_{21}^{\prime}\left(\boldsymbol{\tau}_{1} \times \boldsymbol{\tau}_{2}\right)_{z} \boldsymbol{\sigma}_{1} \times \mathbf{k}_{2}\right] \times \mathbf{q}+1 \rightleftharpoons 2
\end{aligned}
$$

We fix the LECs multiplying the isovector operators by relating them, in a resonance saturation picture, to the couplings of the $N$ to $\Delta$ excitation, i.e.,

$$
\frac{d_{8}^{\prime}}{4} \rightarrow \frac{\mu_{\gamma N \Delta} h_{A}}{9 m_{N}\left(m_{\Delta}-m_{N}\right)}, \quad d_{21}^{\prime}=\frac{d_{8}^{\prime}}{4},
$$

where $m_{\Delta}$ and $h_{A}$ are the mass of the $\Delta\left(m_{\Delta}-m_{N}=\right.$ $294 \mathrm{MeV}$ ) and the $N$ to $\Delta$ axial coupling constant, $\mu_{\gamma N \Delta}=3$ n.m. is the transition magnetic moment [26], and $h_{A} / F_{\pi}=f_{\pi N \Delta} / m_{\pi}$ with $f_{\pi N \Delta}^{2} /(4 \pi) \simeq 0.35$ as obtained by equating the first-order expression of the $\Delta$ decay width with the experimental value. The current proportional to these LECs, $d_{8}^{\prime}$ and $d_{21}^{\prime}$, reduces to the conventional $N-\Delta$ current. The isoscalar term in Eq. (18) saturates the standard $\rho \pi \gamma$ transition current [12, 13, 20].

The EM operators described above have power-law behavior for large momenta and need to be regularized before they can be inserted between nuclear wave functions. The regularization procedure is implemented by means of a cutoff of the form [13]

$$
C_{\Lambda}(k)=\exp \left(-k^{4} / \Lambda^{4}\right)
$$

We utilize the $\chi$ EFT operators within a hybrid context, in which the matrix elements are evaluated with wave functions that are solutions of the realistic Hamiltonian given in Eq. (2). Intrinsic to this approach is a mismatch between the short-range behavior of the nuclear potential and that of the EM operator. As a consequence, the current is not strictly conserved.

The fitting of the unknown LECs entering the EM currents, namely $C_{15}^{\prime}, C_{16}^{\prime}, d_{8}^{\prime}$, and $d_{9}^{\prime}$ (with $d_{21}^{\prime}=d_{8}^{\prime} / 4$, as implied by the $\Delta$-saturation mechanism) was done in Ref. [13]. In that work, the cut-off $\Lambda$ was varied in the range (500-600) MeV and the LECs were constrained to reproduce a set of nuclear EM observables for any given $\Lambda$ in this range. Three different parametrizations were tested in the $A=2-3$ nuclei. The trinucleon wave functions, required for the evaluation of the matrix elements, were obtained with the hyperspherical harmonics (HH) expansion discussed in Refs. 27 29 with a nuclear Hamiltonian consisting of the Argonne $v_{18}$ (AV18) [3] and Urbana IX (UIX) [30] potentials. (There is very little difference between the $A=3$ wave functions for AV18+UIX and AV18+IL7.) The three models (labeled model I, II, and III) determine the LECs multiplying isoscalar operators (i.e. $C_{15}^{\prime}$ and $d_{9}^{\prime}$ ) so as to reproduce the experimental deuteron m.m. and the isoscalar combination of the trinucleon m.m.'s. Models II and III fix the isovector LEC $d_{8}^{\prime}$ by $\Delta$-saturation as indicated in Eq. (19), while $C_{16}^{\prime}$ is constrained so as to reproduce either the $n p$ radiative capture cross section at thermal neutron energies in model II, or the isovector combination of the trinucleon magnetic moments in model III. In model I, $d_{8}^{\prime}$ is left as a free parameter and is constrained, along with $C_{16}^{\prime}$, so as to reproduce both the $n p$ radiative capture cross section and the isovector combination of the trinucleon m.m.'s. As already observed in Ref. [13], model I leads to unnaturally large values for both isovector LECs, severely spoiling the convergence pattern of the chiral expansion. We have, nevertheless, tested all three models (with cutoffs of both 500 and $600 \mathrm{MeV}$ ) in VMC m.m. calculations for $A=3-8$ nuclei, and verified that this pathology-i.e., the lack of convergence - persists, and indeed gets worse, in larger systems. We have therefore disregarded model I, and adopted model III with cut-off $\Lambda=600 \mathrm{MeV}$ in the present study. The parameters entering this model, obtained from the calculations performed in Ref. [13], are listed in Table I. Model III (with $\Lambda=600 \mathrm{MeV}$ ), when tested in VMC calculations, produced the best results for the m.m.'s.

\section{B. SNPA current operator}

The two-body currents in the SNPA formalism have been described in detail most recently in Ref. [10]. These currents are separated into model-independent (MI) and model-dependent (MD) terms. The former (MI) are derived from the $N N$ potential (the AV18 in present case), and their longitudinal components satisfy, by construction, current conservation with it. They contain no free parameters, and their short-range behavior is consistent with that of the potential. The dominant terms, isovector in character, originate from the static part of the potential, which is assumed to be due to exchanges of effective pseudoscalar (PS or " $\pi$-like") and vector (V or " $\rho$-like") mesons. The associated currents are then constructed by using the PS and V propagators, projected out of the static potential [10]. Additional (short-range) currents follow by minimal substitution in the momentumdependent part of the potential. They have both isoscalar and isovector terms, and lead to contributions which are typically much smaller (in magnitude) than those generated by the PS and V currents. At large inter-nucleon separations, where the $N N$ potential is driven by the OPE mechanism, the MI current coincides with the standard seagull and pion-in-flight OPE currents diagrammatically illustrated in panels (b) and (c), respectively, of Fig. 1 .

The MD currents are purely transverse, and unconstrained by current conservation. The dominant term is associated with excitation of intermediate $\Delta$ isobars, which are treated non-perturbatively with the transitioncorrelation-operator method developed in Ref. [31]. These $\Delta$ currents are discussed in considerable detail in 
Ref. 2]. Additional (and numerically small) MD currents arise from the isoscalar $\rho \pi \gamma$ and isovector $\omega \pi \gamma$ transition mechanisms. The values for the coupling constants entering them are also listed in Ref. [2].

\section{RESULTS}

The IA m.m. for the 8- and 9-body nuclei have significantly higher Monte Carlo statistical errors than most quantities that we have computed with GFMC. Therefore we present two examples of the GFMC propagation as a function of the imaginary time $\tau$. Fig. 2 shows the propagation of a typical case, ${ }^{8} \mathrm{Li}$. Three propagations are shown, one in which the constrained propagation [19] is relaxed with 40 unconstrained steps $\left(n_{u}=40\right)$ and two with $n_{u}=80$. The $n_{u}=40$ case was made with 10,000

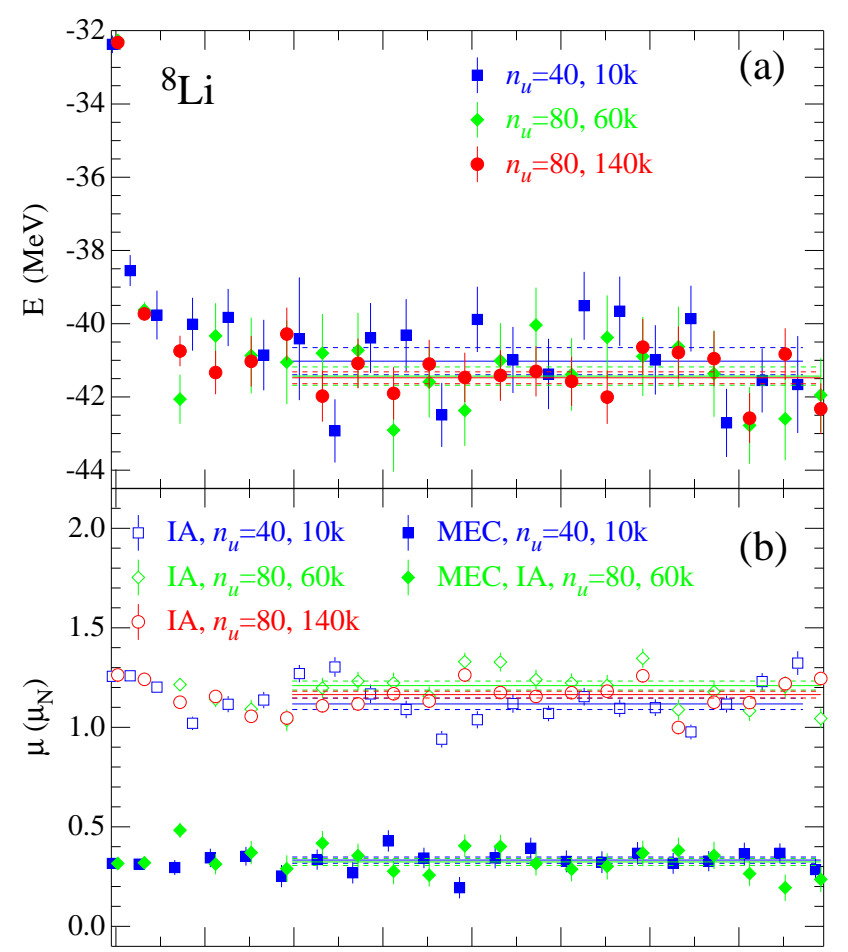

FIG. 2: (Color online) Propagation of the energy (a) and m.m. (b) as a function of imaginary time $\tau$ for the ground state of ${ }^{8} \mathrm{Li}$.

walkers while the $n_{u}=80$ cases are averages of calculations with 20,000 and 40,000 (60,000 total) and 20,000, 40,000 and 80,000 walkers (140,000 total), respectively. The energy is shown in panel (a); it is similar to results shown in Ref. [19]. As can be seen, there is a rapid drop from the initial $\mathrm{VMC}$ value at $\tau=0$ that reaches a stable result before $0.1 \mathrm{MeV}^{-1}$. The results for all quantities presented in this article are averages over $\tau$ from 0.2 to $0.8 \mathrm{MeV}^{-1}$, as indicated by the solid lines, with statistical errors denoted by the dashed lines. The propagations of point proton and neutron radii are similar, except that the starting VMC values are within $5 \%$ of the final results. As has been shown in Ref. [19], these quantities are all converged by $n_{u}=40$, often by $n_{u}=20$. The quadrupole moments are much more difficult to evaluate, because they have long-lived oscillations in the propagation time $\tau$.

The IA and $\chi$ EFT MEC m.m. are shown in panel (b). The statistical fluctuations of the IA term are much worse than those of the MEC term when the same number of configurations are used. Also there may be a small systematic change in the IA term going from $n_{u}=40$ to $n_{u}=80$; the average values are 1.120(27) and 1.164(17), respectively, giving a difference of $0.044(32)$. The MEC does not have this sensitivity; the two calculations are in excellent agreement. However because of the rapid growth of statistical error with increasing $n_{u}$, the $n_{u}=80$ calculation needs seven times as many walkers to achieve the same statistical error.

The Monte Carlo statistical errors in our computed m.m.'s are most severe for ${ }^{9} \mathrm{C}$, as is shown in Fig. 3. Two

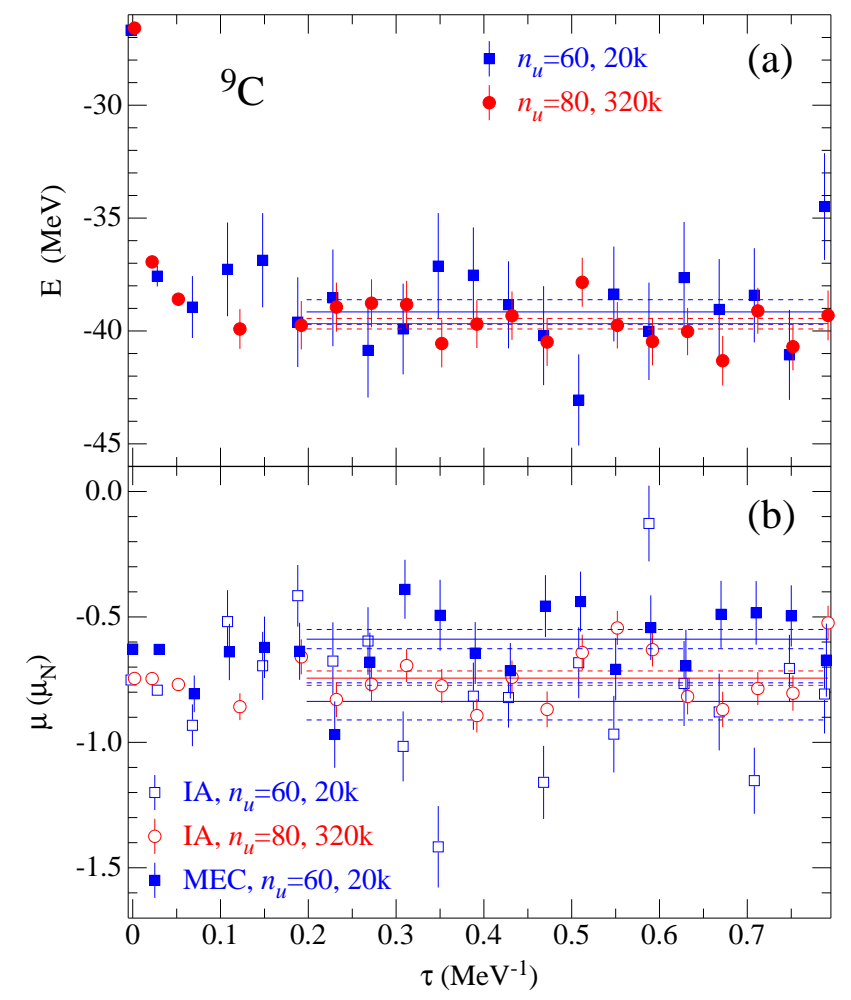

FIG. 3: (Color online) Propagation of the energy (a) and m.m. (b) as a function of imaginary time $\tau$ for the ground state of ${ }^{9} \mathrm{C}$.

propagations are shown, one in with $n_{u}=60$ and one with $n_{u}=80$. The first was made using 20,000 walkers while the latter is the average of two calculations, each with 160,000 walkers. Again the energy, shown in the panel (a), is well converged for both cases. As before for ${ }^{8} \mathrm{Li}$, the $\chi$ EFT MEC m.m., panel (b), has smaller fluctuations for a given number of walkers than the IA m.m. 
In this case the $\chi \mathrm{EFT}$ MEC is a large (80\%) addition to the IA m.m.

In both examples the statistical fluctuations in the IA term are much larger than in the MEC term. However the evaluation of the MEC requires much more computational effort per walker than does the propagation and IA term. Therefore for most of the calculations, we propagate a large number of walkers using $n_{u}=60$ or 80 to obtain the IA m.m. term (and also the other reported quantities). The MEC is obtained with comparable statistical error using fewer walkers and the two numbers and their errors combined to get the total m.m. The propagations are averaged over $\tau=0.2$ to $0.8 \mathrm{MeV}^{-1}$.

The large statistical fluctuations (and possible $n_{u}$ sensitivity) are coming from the IV combination of the spin term of the IA m.m., Eq. (14). The IV convection term and both IS terms have much smaller fluctuations. Thus if isospin symmetry is assumed for the wave functions of isobaric analogs, we can make precise statements about the IS m.m. However if we do not want to assume such isospin symmetry, as in the ${ }^{9} \mathrm{C}-{ }^{9} \mathrm{Li}$ case below, then we have to make separate calculations for each nucleus and the large errors in the IV parts make the extraction of an IS m.m. with small statistical error impossible.

The energies $E$, point proton $\mathrm{rms}$ radii $r_{p}$ (and point neutron rms radii $r_{n}$ for $N \neq Z$ nuclei), m.m.'s $\mu$ in IA, and quadrupole moments $Q$ for the nuclear states calculated in this work are presented in Table Ialong with experimental values where available. Experimental energies are from Ref. 33], EM moments are from Refs. [34 37], and point radii are converted from the charge radii given in Refs. 38 42]. Many energies for $A \leq 7$ nuclei evaluated with the AV18+IL7 Hamiltonian have been reported previously in Ref. 43]. The present energies, which are from independent calculations, are in agreement with the previous results within the Monte Carlo statistical errors shown in parentheses.

For many of the isobaric analog states, the energy and moments are calculated using the GFMC wave functions generated for the $T_{z}=-T$ state and then simply interchanging protons and neutrons to evaluate the $T_{z}=+T$ state. These calculations are denoted by an asterisk $\left(^{*}\right)$ in the table, and will be referred to as chargesymmetry-conserving (CSC) results. For ${ }^{3} \mathrm{He},{ }^{8} \mathrm{~B}$, and ${ }^{9} \mathrm{C}$ ground states we also made independent calculations with different starting VMC wave functions and different isoscalar Coulomb terms [1, 32] in the GFMC propagator appropriate to the $T_{z}=+T$ state. We then use these wave functions to predict the quantities in their isobaric analogs, i.e., ${ }^{3} \mathrm{H},{ }^{8} \mathrm{Li}$, and ${ }^{9} \mathrm{Li}$. The pairs of independent solutions for the isobaric analogs will be referred to as charge-symmetry-breaking (CSB) results. Thus six nuclear states have two entries in the table, comparing a direct calculation with the prediction by charge symmetry from its isobaric analog.

We see from the table that the energies in these paired calculations are in generally good agreement, with the largest discrepancy for $A=9$, where the difference is $\sim 2 \%$ and the statistical errors almost touch. In each of the CSB cases, the $T=-T_{z}$ state is more bound than its isobaric analog, and the expectation values of individual terms in the nuclear Hamiltonian, like $\left\langle K_{i}\right\rangle$ and $\left\langle v_{i j}\right\rangle$, are larger in magnitude.

The point nucleon rms radii are slightly larger for the proton-rich nuclei compared to the charge symmetric solution from the proton-poor isobaric analog. For example, the proton rms radius of ${ }^{9} \mathrm{Li}\left({ }^{9} \mathrm{C}\right)$ is smaller (larger) when the appropriate Coulomb term is included in the GFMC propagator, indicating that the system is more compact (diffuse). If ${ }^{9} \mathrm{Li}\left({ }^{9} \mathrm{C}\right)$ is constructed from the ${ }^{9} \mathrm{C}\left({ }^{9} \mathrm{Li}\right)$ solution, then it appears to be a more diffuse (compact) system. Consistently with this weak sensitivity of the calculated energies and radii to the charge symmetry picture implemented to derive the nuclear wave functions, we find that the calculated m.m.'s in IA are not statistically different in the $T=\frac{1}{2}, 1$ cases and we see only very weak evidence that the IA m.m.'s of the $A=9, T=3 / 2$ systems are affected by charge symmetry. The quadrupole moments are also fairly consistent in the paired results and close to the experimental values.

\section{A. Magnetic Moments in $A=2-9$ Nuclei}

The calculations of the matrix elements, both diagonal and off diagonal, have been described in detail in Refs. 1, 2]. In particular, the IA matrix element is evaluated using the $M 1$ operator induced by the one-body current given in Eq. (14), namely

$$
\boldsymbol{\mu}^{\mathrm{IA}}=\sum_{i}\left(e_{N, i} \mathbf{L}_{i}+\mu_{N, i} \boldsymbol{\sigma}_{i}\right)
$$

The matrix element associated with the MEC contribution is

$$
\begin{aligned}
& \left\langle J_{f}^{\pi}, M_{f}\left|\mu^{\mathrm{MEC}}\right| J_{i}^{\pi}, M_{i}\right\rangle= \\
& -i \lim _{q \rightarrow 0} \frac{2 m_{N}}{q}\left\langle J_{f}^{\pi}, M_{f}\left|j_{y}^{\mathrm{MEC}}(q \hat{\mathbf{x}})\right| J_{i}^{\pi}, M_{i}\right\rangle,
\end{aligned}
$$

where the spin-quantization axis and momentum transfer $\mathbf{q}$ are, respectively, along the $\hat{\mathbf{z}}$ and $\hat{\mathbf{x}}$ axes, and $M_{J}=J$. The various contributions are evaluated for two small values of $q<0.02 \mathrm{fm}^{-1}$ and then extrapolated smoothly to the limit $q=0$. The error due to extrapolation is much smaller than the statistical error in the Monte Carlo sampling.

In Table III we show, in addition to the proton and neutron experimental m.m.'s, the experimental and calculated m.m.'s for the $A=2$ and 3 nuclei, including MEC contributions from the EM currents in the SNPA and $\chi$ EFT models. In the table we label with IS and IV the isoscalar and isovector combinations of the magnetic moments as given by:

$$
\mu\left(T, T_{z}\right)=\mu(\mathrm{IS})+\mu(\mathrm{IV}) T_{z} .
$$


TABLE II: GFMC results for $A \leq 9$ nuclear states studied in this work, compared to experimental values 33 42]. Numbers in parentheses are statistical errors for the GFMC calculations or experimental errors; errors of less than one in the last decimal place are not shown.

\begin{tabular}{|c|c|c|c|c|c|c|c|c|}
\hline \multirow[t]{2}{*}{$\overline{{ }^{A} Z\left(J^{\pi}, T\right)}$} & \multicolumn{2}{|c|}{$E[\mathrm{MeV}]$} & \multicolumn{2}{|c|}{$r_{p}\left[r_{n}\right][\mathrm{fm}]$} & \multicolumn{2}{|c|}{$\mu(\mathrm{IA})$ [n.m.] } & \multicolumn{2}{|c|}{$Q\left[\mathrm{fm}^{2}\right]$} \\
\hline & GFMC & Expt. & GFMC & Expt. & GFMC & Expt. & $\overline{\text { GFMC }}$ & Expt. \\
\hline${ }^{3} \mathrm{H}\left(\frac{1}{2}^{+}, \frac{1}{2}\right)$ & $-8.50(1)$ & -8.482 & $1.58[1.76]$ & $1.58(10)$ & 2.556 & 2.979 & & \\
\hline${ }^{3} \mathrm{He}\left(\frac{1}{2}^{+}, \frac{1}{2}\right)$ & $-7.73(1)$ & -7.718 & $1.80[1.60]$ & $1.76(1)$ & -1.743 & -2.127 & & \\
\hline${ }^{3} \mathrm{He}\left(\frac{1}{2}^{+}, \frac{1}{2}\right) *$ & $-7.75(1)$ & & $1.76[1.58]$ & & -1.750 & & & \\
\hline $\begin{array}{l}{ }^{6} \mathrm{Li}\left(1^{+}, 0\right) \\
{ }^{6} \mathrm{Li}\left(0^{+}, 1\right)\end{array}$ & $\begin{array}{l}-31.82(3) \\
-28.44(4)\end{array}$ & $\begin{array}{l}-31.99 \\
-28.43\end{array}$ & 2.39 & $2.45(4)$ & 0.817 & 0.822 & $-0.20(6)$ & $-0.082(2)$ \\
\hline${ }^{7} \mathrm{Li}\left(\frac{1}{2}^{-}, \frac{1}{2}\right)$ & $-38.9(1)$ & -38.76 & & & & & & \\
\hline${ }^{7} \mathrm{Be}\left(\frac{3}{2}^{-}, \frac{1}{2}\right)^{*}$ & $-37.4(1)$ & -37.60 & $2.47[2.28]$ & $2.51(2)$ & -1.06 & $-1.398(15)$ & $-6.7(1)$ & \\
\hline${ }^{7} \mathrm{Be}\left(\frac{1}{2}^{-}, \frac{1}{2}\right) *$ & $-37.3(1)$ & -37.17 & & & & & & \\
\hline${ }^{8} \mathrm{Li}\left(2^{+}, 1\right)$ & $-41.5(2)$ & -41.28 & $2.10[2.46]$ & $2.20(5)$ & $1.16(2)$ & 1.654 & $3.3(1)$ & $3.14(2)$ \\
\hline${ }^{8} \operatorname{Li}\left(2^{+}, 1\right)^{*}$ & $-41.0(2)$ & & $2.11[2.48]$ & & $1.13(3)$ & & $3.0(4)$ & \\
\hline${ }^{8} \mathrm{~B}\left(3^{+}, 1\right)^{*}$ & $-34.8(3)$ & -35.42 & & & & & & \\
\hline${ }^{9} \mathrm{Be}\left(\frac{3}{2}^{-}, \frac{1}{2}\right)$ & $-58.1(2)$ & -58.16 & $2.37(1)[2.56(1)]$ & $2.38(1)$ & $-1.18(1)$ & -1.178 & $5.1(1)$ & $5.29(4)$ \\
\hline${ }^{9} \mathrm{Be}\left(\frac{5}{2}^{-}, \frac{1}{2}\right)$ & $-55.7(2)$ & -55.74 & & & & & & \\
\hline${ }^{9} \mathrm{~B}\left(\frac{3}{2}^{-}, \frac{1}{2}\right)^{*}$ & $-56.3(1)$ & -56.31 & $2.56(1)[2.37(1)]$ & & $2.97(1)$ & & $4.0(3)$ & \\
\hline${ }^{9} \mathrm{~B}\left(\frac{5}{2}^{-}, \frac{1}{2}\right)^{*}$ & $-53.9(2)$ & -53.95 & & & & & & \\
\hline${ }^{9} \mathrm{Li}\left(\frac{3}{2}^{-}, \frac{3}{2}\right)$ & $-45.2(3)$ & -45.34 & $1.97(1)[2.36(2)]$ & $2.11(5)$ & $2.66(3)$ & 3.437 & $-2.3(1)$ & $-3.06(2)$ \\
\hline${ }^{9} \operatorname{Li}\left(\frac{3}{2}^{-}, \frac{3}{2}\right) *$ & $-45.9(3)$ & & $2.03(1)[2.45(1)]$ & & $2.64(4)$ & & $-2.7(2)$ & \\
\hline${ }^{9} \mathrm{Li}\left(\frac{1}{2}^{-}, \frac{3}{2}\right)$ & $-43.2(4)$ & -42.65 & & & & & & \\
\hline${ }^{9} \mathrm{C}\left(\frac{3}{2}^{-}, \frac{3}{2}\right)$ & $-39.7(3)$ & -39.04 & $2.45(1)[2.03(1)]$ & & $-0.75(3)$ & -1.391 & $-4.1(4)$ & \\
\hline${ }^{9} \mathrm{C}\left(\frac{3}{2}^{-}, \frac{3}{2}\right)^{*}$ & $-38.8(3)$ & & $2.36(2)[1.97(1)]$ & & $-0.82(4)$ & & $-3.7(1)$ & \\
\hline
\end{tabular}

With the label MEC we denote anything that goes beyond the IA picture, therefore the $\chi \mathrm{EFT} \mathrm{MEC}$ current includes also the one-body relativistic correction operator entering at N2LO. The results for the deuteron are from calculations of matrix elements with wave functions which are exact solutions of the two-body Schrödinger equation with the AV18 potential. Results for the $A=3$ nuclei are from GFMC calculations with the nuclear Hamiltonian consisting of the AV18 two-body and IL7 three-body potentials (AV18+IL7), while those designated with $\mathrm{HH}$ are results from hyperspherical harmonics from Ref. [13] obtained with the nuclear Hamiltonian consisting of the AV18 and the UIX three-body potentials (AV18+UIX). Both the GFMC and $\mathrm{HH}$ wave functions have been constructed separately without exploiting charge symmetry. Strictly speaking, the GFMC m.m.'s are for the propagating Hamiltonian $H^{\prime}$, i.e., $\mathrm{AV}^{\prime}+\mathrm{IL}^{\prime}{ }^{\prime}$, as discussed in Sec. II The small $0.3 \%-0.5 \%$ difference between the GFMC and HH IA values may be attributable to this difference in the Hamiltonians.

Numerical differences between the calculated $\chi \mathrm{EFT}$ MEC terms are also affected by an additional approximation implemented in the GFMC calculations. The one-body m.m. operator associated with the relativistic correction at N2LO (illustrated in panel (d) of Fig. 1) reads [11]

$$
\begin{aligned}
\boldsymbol{\mu}^{\mathrm{N} 2 \mathrm{LO}}= & -\frac{e}{8 m_{N}^{3}} \sum_{i=1}^{A}\left[\left\{p_{i}^{2}, e_{N, i} \mathbf{L}_{i}+\mu_{N, i} \boldsymbol{\sigma}_{i}\right\}\right. \\
& \left.+e_{N, i} \mathbf{p}_{i} \times\left(\boldsymbol{\sigma}_{i} \times \mathbf{p}_{i}\right)\right],
\end{aligned}
$$

where $\mathbf{p}_{i}=-i \nabla_{i}$ and $\mathbf{L}_{i}$ are the linear momentum and angular momentum operators of particle $i$, and $\{\ldots, \ldots\}$ denotes the anticommutator. In the GFMC calculations we do not explicitly evaluate the $\mathbf{p}_{i}^{2}$ term, but instead approximate it with its average value, that is $\mathbf{p}_{i}^{2} \sim<\mathbf{p}_{i}^{2}>$, as determined from the expectation value of the kinetic energy operator in each nucleus. This approximation leads, in the case of ${ }^{3} \mathrm{He}$, to a $5 \%$ difference in the MEC correction which itself is a $20 \%$ correction to the total calculated m.m., and to an even smaller effect in ${ }^{3} \mathrm{H}$. The values utilized in the GFMC calculations are reported in Table IV. for the nuclei investigated in this work. Of course, this approximation only affects the N2LO $\chi$ EFT operator. The HH calculations use the nonapproximated operator at N2LO and are designated with 
TABLE III: Magnetic moments in nuclear magnetons for $A=2-3$ nuclei evaluated with SNPA and $\chi$ EFT EM current operators. The current model labeled $\chi \mathrm{EFT}^{\dagger}$ accounts for an exact calculation of the N2LO relativistic correction to the IA current (see text for explanation). Results labeled with $\mathrm{HH}$ are obtained utilizing trinucleon wave functions constructed with the hyperspherical harmonics (HH) method developed in Refs. 27 29], and a nuclear Hamiltonian consisting of AV18+UIX. The remaining results (except for the deuteron ones which are exact) are from GFMC calculations discussed in the text. The spatial symmetry (s.s.) of the nuclear wave function is also given. The IS and IV labels indicate the isoscalar and isovector combinations. Results obtained with the $\chi \mathrm{EFT}$ and $\chi \mathrm{EFT}^{\dagger}$ models are not predictions (see text for explanation).

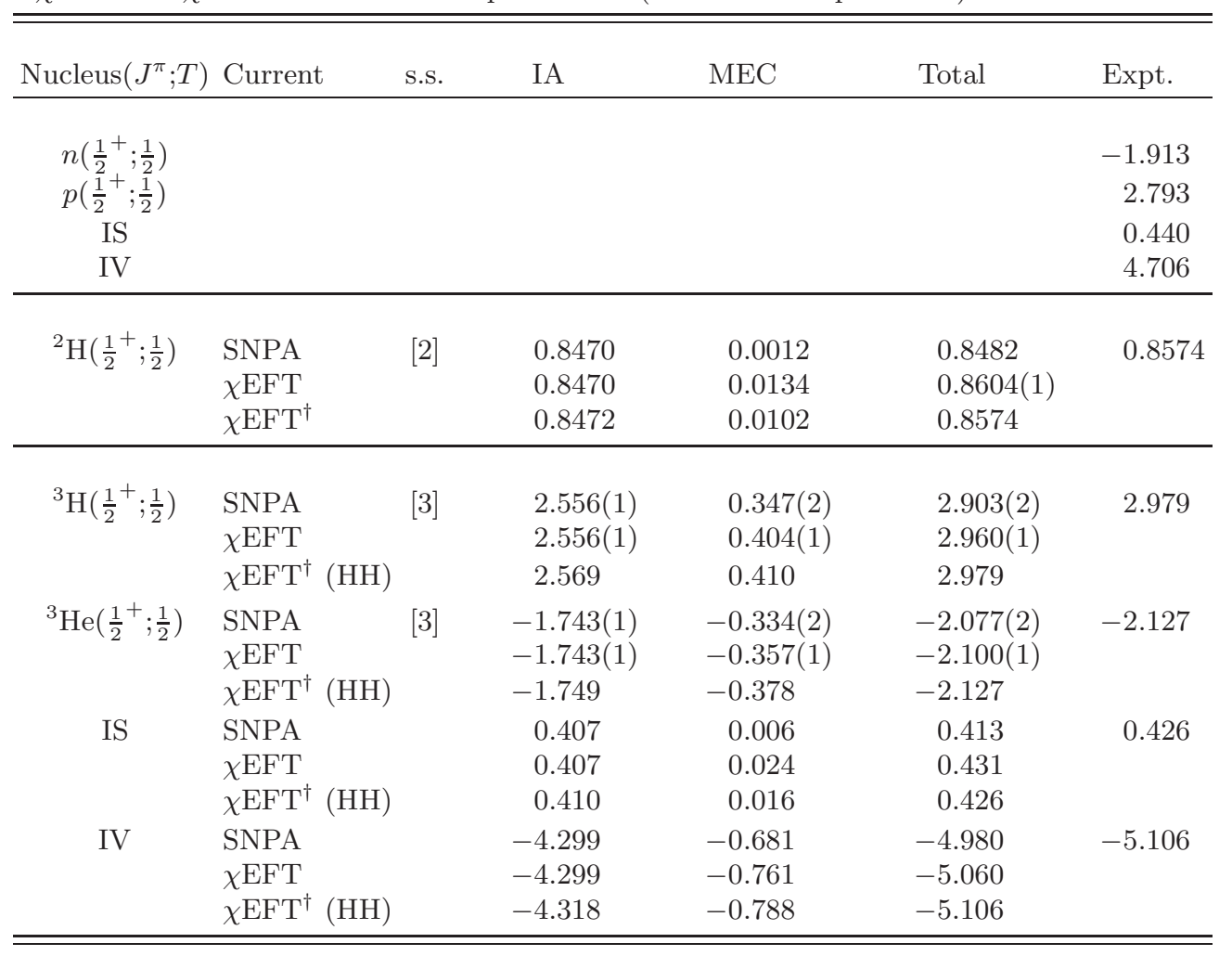

TABLE IV: Values (in units of $\mathrm{fm}^{-2}$ ) of the approximated $\left\langle p_{i}^{2}\right\rangle$, entering the $\chi$ EFT current at N2LO, used in the GFMC calculations.

\begin{tabular}{c|cccccc}
\hline \hline$A$ & 2 & 3 & 6 & 7 & 8 & 9 \\
\hline$\left\langle p_{i}^{2}\right\rangle$ & 0.5 & 0.8 & 1.2 & 1.2 & 1.2 & 1.3 \\
\hline \hline
\end{tabular}

$\chi \mathrm{EFT}^{\dagger}$. These calculations have been used to constrain the LECs entering the $\chi \mathrm{EFT}$ currents, therefore the $\mathrm{HH}$ results presented in Table III are not predictions, in that they reproduce the experimental data by construction.

There are also tiny numerical differences between the SNPA calculations presented here and those reported in Ref. [2]. These may be due to differences in the starting variational wave functions as well as systematic uncertainties in the GFMC calculation. At any rate, all the numerical differences mentioned above are of little importance if one accounts for the sensitivity of these results to the nuclear and current models utilized. The results in the $A=2$ and 3 nuclei show that the SNPA model underestimates the isoscalar component in both the deuteron and the trinucleon m.m.'s.

In Table $\mathrm{V}$, we report the GFMC calculations for the m.m.'s of the $A=6-9$ nuclei. We compare results obtained using either the SNPA or the $\chi$ EFT MEC currents. The MEC corrections evaluated in both models are qualitatively in agreement. They boost the IA in the direction of the experimental data in all cases, except for ${ }^{6} \mathrm{Li}$ and ${ }^{9} \mathrm{Be}$. In these systems the IA results are already in very good agreement with the experimental data while the small MEC contributions make the predictions slightly worse.

The calculations denoted with an asterisk $(*)$ are obtained exploiting charge symmetry, that is by interchanging protons and neutrons to generate the isobaric analogs wave functions. For the $A=7,8$ nuclei, we verified that m.m. predictions obtained with independent nuclear wave functions for the isobaric analogs, i.e., calculations which account for the proper isoscalar Coulomb term in the starting VMC wave functions as well as in the GFMC propagator, are essentially identical to the standard charge symmetric results, and therefore we do not 
TABLE V: Magnetic moments in nuclear magnetons for $A=6-9$ nuclei evaluated with SNPA and $\chi$ EFT EM current operators. Results labeled with a star are obtained exploiting charge symmetry. The dominant spatial symmetry (s.s.) of the nuclear wave function is given.

\begin{tabular}{|c|c|c|c|c|c|c|}
\hline$\overline{\overline{\text { Nucleus }}\left(J^{\pi} ; T\right)}$ & Current & s.s. & $\overline{\mathrm{IA}}$ & $\overline{\mathrm{MEC}}$ & Total & Expt. \\
\hline${ }^{6} \mathrm{Li}\left(1^{+} ; 0\right)$ & $\begin{array}{l}\text { SNPA } \\
\chi \mathrm{EFT}\end{array}$ & {$[42]$} & $\begin{array}{l}0.817(1) \\
0.817(1)\end{array}$ & $\begin{array}{r}-0.010(1) \\
0.020(1)\end{array}$ & $\begin{array}{l}0.807(1) \\
0.837(1)\end{array}$ & $\overline{0.822}$ \\
\hline${ }^{7} \operatorname{Li}\left(\frac{3}{2}^{-} ; \frac{1}{2}\right)$ & $\begin{array}{l}\text { SNPA } \\
\chi \mathrm{EFT}\end{array}$ & {$[43]$} & $\begin{array}{l}2.87(1) \\
2.87(1)\end{array}$ & $\begin{array}{l}0.25(2) \\
0.37(1)\end{array}$ & $\begin{array}{l}3.12(2) \\
3.24(1)\end{array}$ & 3.256 \\
\hline${ }^{7} \mathrm{Be}\left(\frac{3}{2}^{-} ; \frac{1}{2}\right)^{*}$ & $\begin{array}{l}\text { SNPA } \\
\chi \mathrm{EFT}\end{array}$ & {$[43]$} & $\begin{array}{l}-1.06(1) \\
-1.06(1)\end{array}$ & $\begin{array}{l}-0.39(2) \\
-0.36(1)\end{array}$ & $\begin{array}{l}-1.45(2) \\
-1.42(1)\end{array}$ & -1.398 \\
\hline IS & $\begin{array}{l}\text { SNPA } \\
\chi \mathrm{EFT}\end{array}$ & & $\begin{array}{l}0.90 \\
0.90\end{array}$ & $\begin{array}{r}-0.07 \\
0.01\end{array}$ & $\begin{array}{l}0.83 \\
0.91\end{array}$ & 0.929 \\
\hline IV & $\begin{array}{l}\text { SNPA } \\
\chi \mathrm{EFT}\end{array}$ & & $\begin{array}{l}-3.93 \\
-3.93\end{array}$ & $\begin{array}{l}-0.64 \\
-0.73\end{array}$ & $\begin{array}{l}-4.57 \\
-4.66\end{array}$ & -4.654 \\
\hline${ }^{8} \mathrm{Li}\left(2^{+} ; 1\right)$ & $\begin{array}{l}\text { SNPA } \\
\chi \mathrm{EFT}\end{array}$ & {$[431]$} & $\begin{array}{l}1.16(2) \\
1.16(2)\end{array}$ & $\begin{array}{l}0.20(2) \\
0.33(1)\end{array}$ & $\begin{array}{l}1.36(3) \\
1.49(2)\end{array}$ & 1.654 \\
\hline${ }^{8} \mathrm{~B}\left(2^{+} ; 1\right)^{*}$ & $\begin{array}{l}\text { SNPA } \\
\chi \mathrm{EFT}\end{array}$ & [431] & $\begin{array}{l}1.42(2) \\
1.42(2)\end{array}$ & $\begin{array}{l}-0.42(2) \\
-0.31(1)\end{array}$ & $\begin{array}{l}1.00(3) \\
1.11(2)\end{array}$ & 1.036 \\
\hline IS & $\begin{array}{l}\text { SNPA } \\
\chi \mathrm{EFT}\end{array}$ & & $\begin{array}{l}1.29 \\
1.29\end{array}$ & $\begin{array}{r}-0.11 \\
0.01\end{array}$ & $\begin{array}{l}1.18 \\
1.30\end{array}$ & 1.345 \\
\hline IV & $\begin{array}{l}\text { SNPA } \\
\chi \mathrm{EFT}\end{array}$ & & $\begin{array}{l}0.13 \\
0.13\end{array}$ & $\begin{array}{l}-0.31 \\
-0.32\end{array}$ & $\begin{array}{l}-0.18 \\
-0.19\end{array}$ & -0.309 \\
\hline${ }^{9} \mathrm{Li}\left(\frac{3}{2}^{-} ; \frac{3}{2}\right)$ & $\begin{array}{l}\text { SNPA } \\
\chi \mathrm{EFT}\end{array}$ & {$[432]$} & $\begin{array}{l}2.66(3) \\
2.66(3)\end{array}$ & $\begin{array}{l}0.34(4) \\
0.70(2)\end{array}$ & $\begin{array}{l}3.00(5) \\
3.36(4)\end{array}$ & 3.437 \\
\hline${ }^{9} \mathrm{C}\left(\frac{3}{2}^{-} ; \frac{3}{2}\right)$ & $\begin{array}{l}\text { SNPA } \\
\chi \mathrm{EFT}\end{array}$ & {$[432]$} & $\begin{array}{l}-0.75(3) \\
-0.75(3)\end{array}$ & $\begin{array}{l}-0.48(4) \\
-0.60(3)\end{array}$ & $\begin{array}{l}-1.23(5) \\
-1.35(4)\end{array}$ & -1.391 \\
\hline IS & $\begin{array}{l}\text { SNPA } \\
\chi \mathrm{EFT}\end{array}$ & & $\begin{array}{l}0.96 \\
0.96\end{array}$ & $\begin{array}{r}-0.07 \\
0.05\end{array}$ & $\begin{array}{l}0.89 \\
1.01\end{array}$ & 1.023 \\
\hline IV & $\begin{array}{l}\text { SNPA } \\
\chi \mathrm{EFT}\end{array}$ & & $\begin{array}{l}-1.14 \\
-1.14\end{array}$ & $\begin{array}{l}-0.27 \\
-0.43\end{array}$ & $\begin{array}{l}-1.41 \\
-1.57\end{array}$ & -1.609 \\
\hline${ }^{9} \mathrm{Be}\left(\frac{3}{2}^{+} ; \frac{1}{2}\right)$ & $\begin{array}{l}\mathrm{SNPA} \\
\chi \mathrm{EFT}\end{array}$ & {$[441]$} & $\begin{array}{l}-1.18(1) \\
-1.18(1)\end{array}$ & $\begin{array}{l}-0.12(1) \\
-0.11(1)\end{array}$ & $\begin{array}{l}-1.30(1) \\
-1.29(1)\end{array}$ & -1.178 \\
\hline${ }^{9} \mathrm{~B}\left(\frac{3}{2}^{+} ; \frac{1}{2}\right)^{*}$ & $\begin{array}{l}\text { SNPA } \\
\chi \mathrm{EFT}\end{array}$ & [441] & $\begin{array}{l}2.97(1) \\
2.97(1)\end{array}$ & $\begin{array}{r}-0.10(1) \\
0.09(1)\end{array}$ & $\begin{array}{l}2.87(1) \\
3.06(1)\end{array}$ & n.a. \\
\hline IS & $\begin{array}{l}\text { SNPA } \\
\chi \mathrm{EFT}\end{array}$ & & $\begin{array}{l}0.89 \\
0.89\end{array}$ & $\begin{array}{l}-0.11 \\
-0.01\end{array}$ & $\begin{array}{l}0.78 \\
0.88\end{array}$ & \\
\hline IV & $\begin{array}{l}\text { SNPA } \\
\chi \mathrm{EFT}\end{array}$ & & $\begin{array}{l}4.15 \\
4.15\end{array}$ & $\begin{array}{l}0.02 \\
0.20\end{array}$ & $\begin{array}{l}4.17 \\
4.35\end{array}$ & \\
\hline
\end{tabular}

report them. In the $A=9, T=\frac{3}{2}$ case there is weak evidence for a CSB effect so in this case we show the results of the two independent calculations.

Also in the $A=6-9$ nuclear m.m.'s, the difference between the SNPA and $\chi \mathrm{EFT}$ corrections is more pronounced in the isoscalar component. In all cases, the $\chi \mathrm{EFT}$ corrections are more positive (or less negative) than the corresponding SNPA. This makes the $\chi \mathrm{EFT}$ predictions closer to the experimental values. The isovector corrections evaluated with the two models are reasonably in agreement with each other, although they are bigger when derived from the $\chi \mathrm{EFT}$ model. MEC corrections are crucial to bring the theory closer to the experimental values. Their effect is particularly pronounced in the isovector combination of the $A=9, T=3 / 2$ nuclei's m.m.'s, for which the MEC SNPA ( $\chi$ EFT) correction provides $\sim 20 \%(\sim 30 \%)$ of the total calculated isovector contribution.

It is interesting to note that, despite the large effect observed in the $A=9, T=3 / 2$ systems, MEC corrections are considerably smaller in the $A=9, T=1 / 2$ nuclei. This feature can be explained by considering the dominant spatial symmetry (s.s.) of the wave functions associated with the $A=9$ systems. In particular, the dominant spatial symmetry of ${ }^{9} \mathrm{Be}\left({ }^{9} \mathrm{~B}\right)$ is [441], corresponding to an $[\alpha, \alpha, n(p)]$ structure as shown in Ref. [44]. A single nucleon outside an $\alpha$ particle feels no net OPE potential, and this holds true also for a single nucleon outside a double- $\alpha$ [44] symmetry state. Consequently, the NLO OPE currents illustrated in panels (b) and (c) of Fig. 1, which are generally the largest MEC terms in both SNPA and $\chi$ EFT approaches, do not contribute significantly. On the other hand, the dominant spatial symmetry of ${ }^{9} \mathrm{C}\left({ }^{9} \mathrm{Li}\right)$ is $[432] \sim\left[\alpha,{ }^{3} \mathrm{He}\left({ }^{3} \mathrm{H}\right), p p(n n)\right]$, 


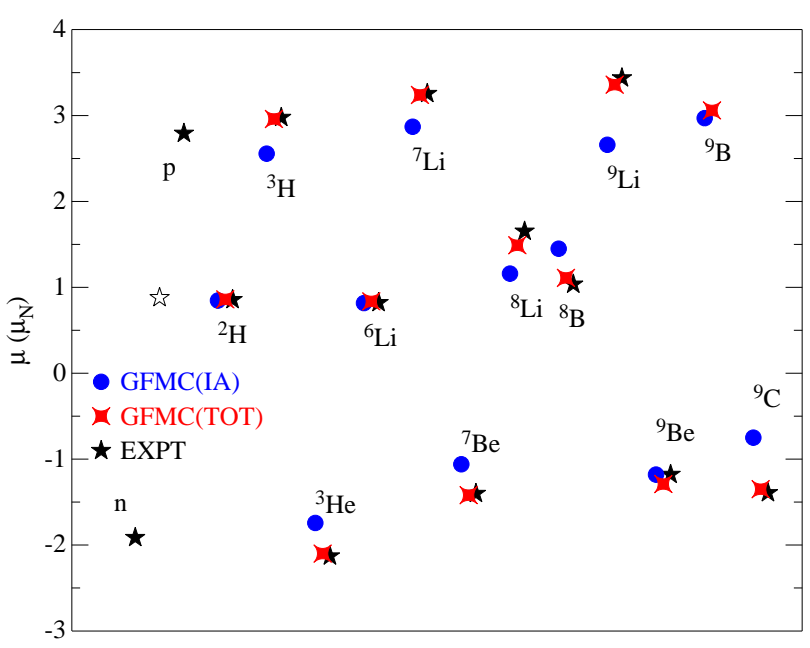

FIG. 4: (Color online) Magnetic moments in nuclear magnetons for $A \leq 9$ nuclei. Black stars indicate the experimental values 35 37., while blue dots (red diamonds) represent GFMC calculations which include the IA one-body EM current (total $\chi \mathrm{EFT}$ current up to N3LO). Predictions are for nuclei with $A>3$.

and the NLO OPE term contributes in both the trinucleon clusters and in between the trinucleon clusters and the valence $p p(n n)$ pair. The IA m.m. for ${ }^{9} \mathrm{Be}$ is close to the experimental value, while those for ${ }^{9} \mathrm{Li}$ and ${ }^{9} \mathrm{C}$ are far from the data, so this pattern of small and large MEC corrections provides good overall agreement with the data.

The $\chi$ EFT results reported in Tables III and V] are summarized in Fig. 4, where the experimental data 3437] (there are no data for the m.m. of ${ }^{9} \mathrm{~B}$ ) are represented by black stars. We show also the experimental values for the proton and neutron m.m.'s, as well as their sum, which corresponds to the m.m. of an Swave deuteron. The experimental values of the $A=2-3$ m.m.'s have been utilized to fix the LECs, therefore predictions are for $A>3$ nuclei. The blue dots labeled as GFMC(IA) represent theoretical predictions obtained with the standard IA one-nucleon EM current entering at LO: diagram (a) of Fig. 1] The GFMC(IA) results reproduce the bulk properties of the m.m.'s of the light nuclei considered here. In particular, we can recognize three classes of nuclei with non-zero m.m.'s, i.e., oddeven nuclei whose m.m.'s are driven by an unpaired valence proton, even-odd nuclei driven by an unpaired valence neutron, and odd-odd nuclei with either a deuteron cluster or a triton-neutron $\left({ }^{3} \mathrm{He}\right.$-proton $)$ cluster outside an even-even core. Predictions which include all the contributions to the N3LO $\chi$ EFT EM currents illustrated in Fig. 1 are represented by the red diamonds of Fig. 4 labeled GFMC(TOT). In all cases except ${ }^{6} \mathrm{Li}$ and ${ }^{9} \mathrm{Be}$ (where the IA is already very good and the MEC correction is very small) the predicted m.m.'s are closer to the experimental data when the MEC corrections are added to the IA one-body EM operator.
It is also interesting to consider the spatial distribution of the various contributions to the m.m., i.e., to examine the magnetic density. The one-body IA contributions from the starting VMC wave functions are shown in Fig. 5 for the isobaric analog pairs ${ }^{7} \mathrm{Li}-{ }^{7} \mathrm{Be},{ }^{8} \mathrm{Li}-{ }^{8} \mathrm{~B}$, and ${ }^{9} \mathrm{Li}-$ ${ }^{9} \mathrm{C}$. (The VMC values for the IA m.m.'s are within a few $\%$ of the final GFMC values, so we expect their spatial distribution to be reasonably accurate.) In the figure, the red upward-pointing triangles are the contribution from the proton spin, $\mu_{p}\left[\rho_{p \uparrow}(r)-\rho_{p \downarrow}(r)\right]$, and similarly the blue downward-pointing triangles are the contribution from the neutron spin. The green diamonds are the proton orbital (convection current) contribution, and the black circles are the sum. The integrals of the black curves over $d^{3} r$ give the total m.m.'s of the nuclei in IA.

For the neutron-rich lithium isotopes, there is one unpaired proton (embedded in a $p$-shell triton cluster) with essentially the same large positive contribution in all three cases. The proton orbital term is also everywhere positive, but relatively small. For ${ }^{7} \mathrm{Li}$ and ${ }^{9} \mathrm{Li}$, the neutrons are paired up, and give only a small contribution, so the total m.m. is close to the sum of the proton spin and orbital parts. However ${ }^{8} \mathrm{Li}$ has one unpaired neutron which acts against the proton and significantly reduces the overall m.m. values. For the proton-rich isobaric analogs, there is one unpaired neutron (embedded in a $p$-shell ${ }^{3}$ He cluster) with the same sizable negative contribution in all three cases. In ${ }^{7} \mathrm{Be}$ and ${ }^{9} \mathrm{C}$, the protons are paired up and give little net contribution, but the orbital term is always positive and acts against the neutron spin term. In ${ }^{8} \mathrm{~B}$ there is also one unpaired proton, which gives a bigger contribution than the unpaired neutron and results in a net positive m.m. value.

In Table VI, we explicitly show the various contributions entering the $\chi \mathrm{EFT}$ operator. The labeling in the table has been defined in Sec. IIIA. We list the contributions at each order. At N3LO, we separate the terms that do not depend on EM LECs (i.e. the LOOP contribution and the contact MIN currents; the former depends on the known axial coupling constant, $g_{A}$, and pion decay amplitude, $F_{\pi}$, while the latter depends on the strong LECs entering the $N N \chi$ EFT potential at N2LO) and those that depend on them (i.e. the contact NM and the OPE current whose isovector component has been saturated with the $\Delta$ transition current). In most cases, chiral convergence is observed but for the isovector N3LO OPE contribution whose order of magnitude is in some cases comparable to the OPE contribution at NLO. It is likely that the explicit inclusion of $\Delta$ degrees of freedom in the present $\chi$ EFT would significantly improve the convergence pattern, since in such a theory this isovector OPE current, presently entering at N3LO, would be promoted to N2LO.

In Table VI we do not provide the errors associated with the individual terms at each order because they are highly correlated. We limit ourselves to report the errors associated with the IA, MEC, and total results. Also in this table, we denote calculations performed enforcing 


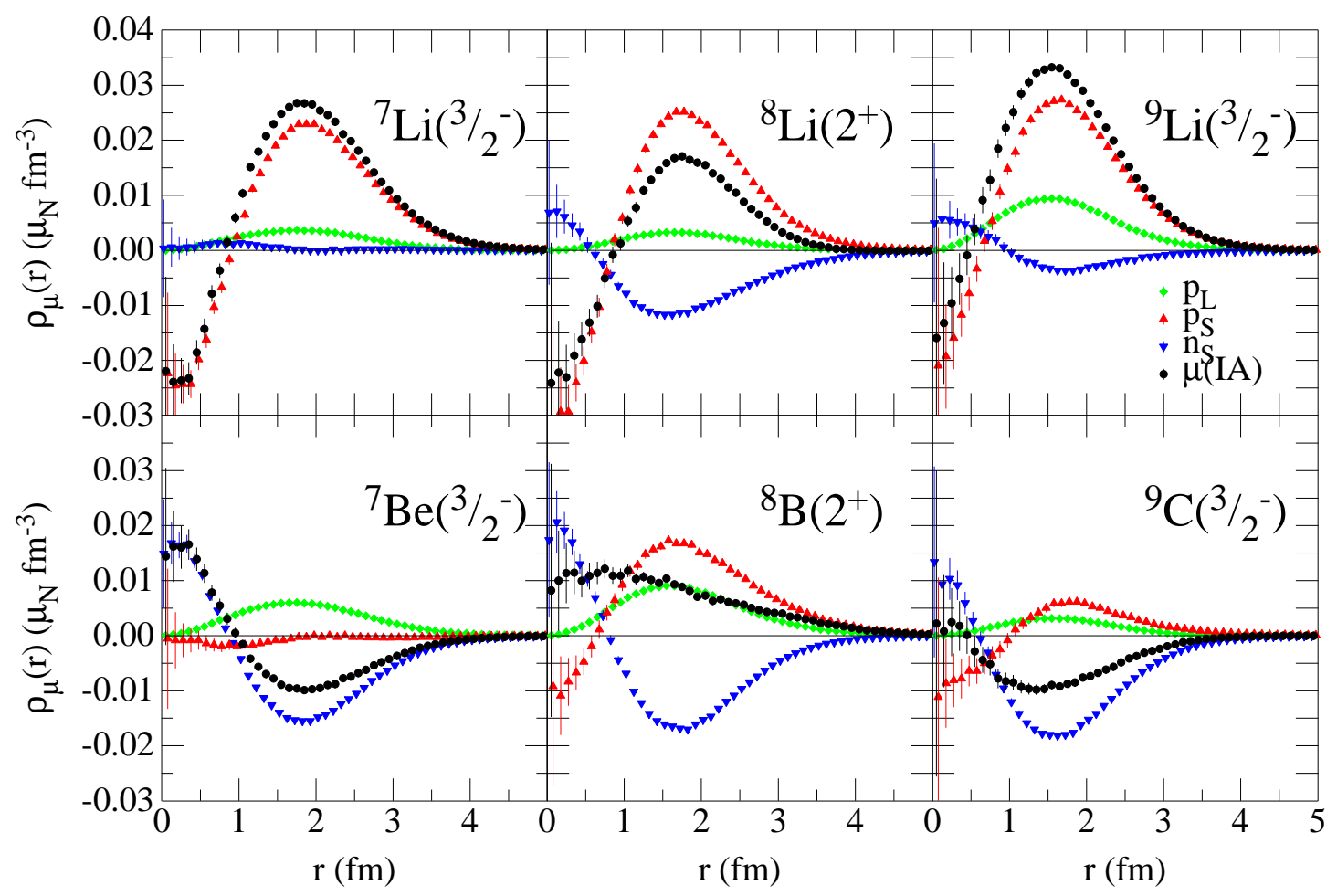

FIG. 5: (Color online) Magnetic density in nuclear magnetons per $\mathrm{fm}^{3}$ for selected nuclei (see text for explanation).

charge symmetry with an asterisk $\left(^{*}\right)$. In these calculations, the isoscalar component of pure isovector operators (that is the OPE operator at NLO and the LOOP operator at N3LO) are obviously zero. Calculations in which the nuclear wave functions are constructed independently present an isoscalar admixture even in the purely isovector corrections, namely the NLO and N3LO LOOP. We do not report the individual contributions entering the SNPA calculations. For the $A \leq 7$ nuclei, they are found to be in agreement with those reported in Ref. [2].

The $A=9, T=3 / 2$ nuclei are very interesting systems not only for the large effect produced by the isovector MEC correction, but also for the 'anomaly' associated with their isoscalar component. In Ref. [45] and references therein, the role of mirror symmetry - or charge symmetry - in the $A=9, T=3 / 2$ nuclei has been investigated. In particular, if mirror symmetry is assumed, that is if the equality of $n n$ and $p p$ forces is enforced, then the isoscalar spin expectation value $\left\langle\sigma_{z}\right\rangle$ can be deduced as

$$
<\sigma_{z}>=\frac{\mu\left(T_{z}=+T\right)+\mu\left(T_{z}=-T\right)-J}{\left(g_{s}^{p}+g_{s}^{n}-1\right) / 2}=\frac{2 \mu(\mathrm{IS})-J}{0.3796} .
$$

In the equation above, $\mu$ and $\mu$ (IS) are the total m.m. and the isoscalar combination of the mirror nuclei m.m.'s, $J$ is the total angular momentum and $g_{s}^{p(n)}$ is the spin $g$ factor of the proton (neutron). The experimental value of $\left\langle\sigma_{z}\right\rangle$ is 1.44 [46] which is - quoting from Ref. [45]"anomalously large if compared to the single-particle es- timate of $1 . "$

Driven by the discussion reported in Ref. [45], we calculate the $\left\langle\sigma_{z}\right\rangle$ value for the $A=9$, $T=3 / 2 \mathrm{nu}-$ clei using the CSB and CSC models described at the beginning of this section. To make this last comment explicit, we show three different calculations for the ${ }^{9} \mathrm{Li}$ and ${ }^{9} \mathrm{C}$ m.m.'s in Table VII using the $\chi$ EFT MEC. The first calculation is performed by constructing the nuclear wave functions independently including the appropriate Coulomb term in the GFMC propagator for each nucleus. The second (third) CSC calculation uses a ${ }^{9} \mathrm{C}\left({ }^{9} \mathrm{Li}\right)$ wave function which is constructed from that of ${ }^{9} \mathrm{Li}\left({ }^{9} \mathrm{C}\right)$ by interchanging protons with neutrons, i.e., by imposing mirror symmetry. The CSB isoscalar component is obtained combining the m.m.'s of ${ }^{9} \mathrm{C}$ and ${ }^{9} \mathrm{Li}$ obtained independently. Therefore the error associated with this observable follows from propagating the statistical errors of the calculated m.m.'s of the $A=9, T=3 / 2$ nuclei. Within the CSC picture, the error associated with the isoscalar combination of the $A=9, T=3 / 2$ m.m.'s can be directly evaluated in the GFMC calculation. These CSC isoscalar values are found to be very stable, with a statistical error of less that $\sim 1 \%$.

We use the CSC and CSB IA and total values for the isoscalar combination of the ${ }^{9} \mathrm{C}$ and ${ }^{9} \mathrm{Li} \mathrm{m} . \mathrm{m}$.'s to evaluate the $\left\langle\sigma_{z}\right\rangle$ value as given in Eq. (25). The results are shown in Table VIII. From this Table, we see that the CSC values are consistent with the single particle prediction of 1 . We observe that, in the CSB calculation both the isoscalar IA and MEC corrections are larger 
TABLE VI: Magnetic moments in nuclear magnetons of $A \leq 9$ nuclei evaluated with the $\chi$ EFT current operator. Individual contributions entering at LO (IA), NLO, N2LO, N3LO are shown (see text for explanation). Remaining notation is as defined in Table $\mathrm{V}$

\begin{tabular}{|c|c|c|c|c|c|c|c|c|c|c|c|}
\hline \multirow[t]{2}{*}{$\operatorname{Nucleus}\left(J^{\pi} ; T\right)$} & \multirow[t]{2}{*}{ s.s. } & \multirow[t]{2}{*}{ IA } & \multirow[t]{2}{*}{ NLO } & \multirow[t]{2}{*}{$\mathrm{N} 2 \mathrm{LO}$} & \multicolumn{2}{|c|}{ N3LO } & \multicolumn{2}{|c|}{ N3LO-LECs } & \multirow[t]{2}{*}{ MEC } & \multirow[t]{2}{*}{ Total } & \multirow[t]{2}{*}{ Expt. } \\
\hline & & & & & $\overline{\text { LOOP }}$ & MIN & NM & OPE & & & \\
\hline${ }^{2} \mathrm{H}\left(1^{+} ; 0\right)$ & {$[2]$} & 0.8470 & - & -0.0042 & - & 0.0364 & -0.0135 & -0.0052 & 0.0135 & 0.8604 & 0.8574 \\
\hline${ }^{3} \mathrm{H}\left(\frac{1}{2}^{+} ; \frac{1}{2}\right)$ & {$[3]$} & $2.556(1)$ & 0.253 & -0.033 & 0.058 & 0.035 & -0.011 & 0.102 & 0.404 & $2.960(1)$ & 2.979 \\
\hline${ }^{3} \mathrm{He}\left(\frac{1}{2}^{+} ; \frac{1}{2}\right)$ & {$[3]$} & $-1.743(1)$ & -0.248 & 0.024 & -0.055 & 0.056 & -0.022 & -0.110 & -0.357 & $-2.100(1)$ & -2.127 \\
\hline IS & & $0.407(1)$ & 0.002 & -0.005 & 0.001 & 0.046 & -0.017 & -0.004 & 0.023 & $0.430(1)$ & 0.426 \\
\hline IV & & $4.299(1)$ & -0.501 & 0.057 & -0.113 & 0.020 & -0.011 & -0.213 & -0.760 & $-5.060(1)$ & -5.106 \\
\hline${ }^{6} \mathrm{Li}\left(1^{+} ; 0\right)$ & {$[42]$} & $0.817(1)$ & - & -0.012 & - & 0.063 & -0.023 & -0.007 & $0.020(1)$ & $0.837(1)$ & 0.822 \\
\hline${ }^{7} \operatorname{Li}\left(\frac{3}{2}^{-} ; \frac{1}{2}\right)$ & {$[43]$} & $2.87(1)$ & 0.237 & -0.062 & 0.064 & 0.034 & -0.012 & 0.107 & $0.37(1)$ & $3.24(1)$ & 3.256 \\
\hline${ }^{7} \mathrm{Be}\left(\frac{3}{2}^{-} ; \frac{1}{2}\right) *$ & {$[43]$} & $-1.06(1)$ & -0.237 & 0.015 & -0.064 & 0.072 & -0.027 & -0.120 & $-0.36(1)$ & $-1.42(1)$ & -1.398 \\
\hline IS & & 0.90 & - & -0.024 & - & 0.053 & -0.020 & -0.006 & 0.01 & 0.91 & 0.929 \\
\hline IV & & -3.93 & -0.473 & 0.078 & -0.127 & 0.038 & -0.014 & -0.227 & -0.73 & -4.66 & -4.654 \\
\hline${ }^{8} \mathrm{Li}\left(2^{+} ; 1\right)$ & {$[431]$} & $1.16(2)$ & 0.226 & -0.038 & 0.045 & 0.056 & -0.021 & 0.062 & $0.33(1)$ & $1.49(2)$ & 1.654 \\
\hline${ }^{8} \mathrm{~B}\left(2^{+} ; 1\right)^{*}$ & {$[431]$} & $1.42(2)$ & -0.226 & -0.020 & -0.045 & 0.090 & -0.033 & -0.077 & $-0.31(1)$ & $1.11(2)$ & 1.036 \\
\hline IS & & 1.29 & - & -0.029 & - & 0.073 & -0.027 & -0.007 & 0.01 & 1.30 & 1.345 \\
\hline IV & & 0.13 & -0.226 & 0.009 & -0.045 & 0.017 & -0.006 & -0.070 & -0.32 & -0.19 & -0.309 \\
\hline${ }^{9} \operatorname{Li}\left(\frac{3}{2}^{-} ; \frac{3}{2}\right)$ & {$[432]$} & $2.66(3)$ & 0.403 & -0.076 & 0.141 & 0.108 & -0.016 & 0.141 & $0.70(2)$ & $3.36(4)$ & 3.437 \\
\hline${ }^{9} \mathrm{C}\left(\frac{3}{2}^{-} ; \frac{3}{2}\right)$ & {$[432]$} & $-0.75(3)$ & -0.372 & 0.039 & -0.135 & 0.058 & -0.031 & -0.156 & $-0.60(3)$ & $-1.35(4)$ & -1.391 \\
\hline IS & & 0.96 & 0.015 & -0.019 & 0.003 & 0.083 & -0.024 & -0.008 & 0.05 & 1.01 & 1.023 \\
\hline IV & & -1.14 & -0.258 & 0.038 & -0.092 & -0.017 & -0.005 & -0.099 & -0.43 & -1.57 & -1.609 \\
\hline${ }^{9} \mathrm{Be}\left(\frac{3}{2}^{+} ; \frac{1}{2}\right)$ & {$[441]$} & $-1.18(1)$ & -0.084 & 0.019 & -0.037 & 0.041 & -0.009 & -0.038 & $-0.11(1)$ & $-1.29(1)$ & -1.178 \\
\hline${ }^{9} \mathrm{~B}\left(\frac{3}{2}^{+} ; \frac{1}{2}\right)^{*}$ & {$[441]$} & $2.97(1)$ & 0.084 & -0.057 & 0.037 & -0.005 & -0.004 & 0.032 & $0.09(1)$ & $3.06(1)$ & n.a. \\
\hline IS & & 0.89 & - & -0.019 & - & 0.018 & -0.007 & -0.003 & -0.01 & 0.88 & \\
\hline IV & & 4.15 & 0.168 & -0.076 & 0.074 & -0.046 & 0.005 & 0.070 & 0.20 & 4.35 & \\
\hline
\end{tabular}

than those obtained in the mirror symmetry based picture. However, the error associated with this last calculation does not allow us conclude with certainty that implementing a CSB picture would resolve the aforementioned 'anomaly' associated with the $A=9, T=3 / 2 \mathrm{nu}$ clei. Within the statistics available at present, we can argue that, in order to reproduce the experimental $\left\langle\sigma_{z}\right\rangle$ value, one would have to combine both the effect of CSB and the correction due to the MEC currents. As briefly mentioned before, we investigated the role of mirror symmetry breaking also in the $A=7,8$ nuclei and found that the calculated m.m.'s are not sensitive to the different nuclear wave functions. A trivial argument to justify this result is to be found in the isospin of the investigated nuclei: intuitively, one would expect the effect of mirror symmetry breaking to increase as $\Delta T_{z}=2 T$ of the mirror nuclei becomes larger.

\section{B. Electromagnetic Transitions in $A=6-9$ Nuclei}

In Table IX, we report the results for the $M 1$ transition matrix elements and the transition widths in nuclei with mass number $A \leq 9$. For these calculations-obtained with the $\chi \mathrm{EFT}$ EM current operator - we report only the IA and the MEC contributions. Experimental data in the table are taken from Refs. 35, 36]. The widths $\Gamma$ in units of $\mathrm{MeV}$ are calculated as

$$
\Gamma_{M 1}=0.890\left(\frac{\Delta E}{\hbar c}\right)^{3} B(M 1)
$$


TABLE VII: Magnetic moments in nuclear magnetons of the $A=9, T=3 / 2$ systems. The nuclear wave functions are derived within a CSB picture (first block), and in a CSC framework (last two blocks). See text for further explanations.

\begin{tabular}{lccrr}
\hline \hline & & & \\
Nucleus $\left(J^{\pi} ; T\right)$ & IA & MEC & Total & Expt. \\
\hline${ }^{9} \mathrm{Li}\left(\frac{3}{2}^{-} ; \frac{3}{2}\right)$ & $2.66(3)$ & $0.70(2)$ & $3.36(4)$ & 3.437 \\
${ }^{9} \mathrm{C}\left(\frac{3}{2}^{-} ; \frac{3}{2}\right)$ & $-0.75(3)$ & $-0.60(3)$ & $-1.35(4)$ & -1.391 \\
$\mathrm{IS}$ & 0.96 & 0.05 & 1.01 & 1.023 \\
$\mathrm{IV}$ & -1.14 & -0.43 & -1.57 & -1.609 \\
\hline \multicolumn{5}{c}{} \\
${ }^{9} \mathrm{Li}\left(\frac{3}{2}^{-} ; \frac{3}{2}\right)$ & $2.66(3)$ & $0.70(2)$ & $3.36(4)$ & 3.437 \\
${ }^{9} \mathrm{C}\left(\frac{3}{2}^{-} ; \frac{3}{2}\right) *$ & $-0.82(4)$ & $-0.68(2)$ & $-1.50(4)$ & -1.391 \\
$\mathrm{IS}$ & 0.93 & 0.01 & 0.94 & 1.023 \\
$\mathrm{IV}$ & -1.16 & -0.46 & -1.62 & -1.609 \\
\hline${ }^{9} \mathrm{Li}\left(\frac{3}{2}^{-} ; \frac{3}{2}\right)^{*}$ & $2.64(4)$ & $0.62(3)$ & $3.26(5)$ & 3.437 \\
${ }^{9} \mathrm{C}\left(\frac{3}{2}^{-} ; \frac{3}{2}\right)$ & $-0.75(3)$ & $-0.60(3)$ & $-1.35(4)$ & -1.391 \\
$\mathrm{IS}$ & 0.94 & 0.01 & 0.95 & 1.023 \\
$\mathrm{IV}$ & -1.13 & -0.40 & -1.53 & -1.609 \\
\hline \hline
\end{tabular}

TABLE VIII: Spin expectation value $\left\langle\sigma_{z}\right\rangle$ for the mirror nuclei ${ }^{9} \mathrm{Li}$ and ${ }^{9} \mathrm{C}$ evaluated within $\mathrm{CSB}$ and CSC frameworks. (See text for explanation).

\begin{tabular}{l|lll}
\hline \hline \multicolumn{1}{c}{ Symmetry } & \multicolumn{1}{c}{ IA } & \multicolumn{1}{c}{ Total } & Expt. \\
\hline $\mathrm{CSB}:{ }^{9} \mathrm{Li}\left(\frac{3}{2}^{-} ; \frac{3}{2}\right),{ }^{9} \mathrm{C}\left(\frac{3}{2}^{-} ; \frac{3}{2}\right)$ & $1.11(11)$ & $1.37(15)$ & 1.44 \\
$\mathrm{CSC}:{ }^{9} \mathrm{Li}\left(\frac{3}{2}^{-} ; \frac{3}{2}\right),{ }^{9} \mathrm{C}\left(\frac{3}{2}^{-} ; \frac{3}{2}\right) *$ & $0.95(1)$ & $1.00(1)$ & \\
$\mathrm{CSC}:{ }^{9} \mathrm{Li}\left(\frac{3}{2}^{-} ; \frac{3}{2}\right)^{*},{ }^{9} \mathrm{C}\left(\frac{3}{2}^{-} ; \frac{3}{2}\right)$ & $1.00(1)$ & $1.05(1)$ & \\
\hline \hline
\end{tabular}

where $\hbar c$ is in units of $\mathrm{MeV}$ fm, and $B(M 1)$ is the squared reduced matrix element of the m.m. operator between the initial and the final nuclear state divided by $\left(2 J_{i}+\right.$ 1 ), with $J_{i}$ the initial state angular momentum. In the equation above, $\Delta E$ is the energy difference between the final and the initial state (in units of $\mathrm{MeV}$ ) for which we take the experimental values as given in Refs. [35, 36].

These calculations are obtained, as before, by propagating up to $\tau=0.8 \mathrm{MeV}^{-1}$ with an evaluation after every 40 propagation steps, i.e., at intervals of $\tau=0.02$ $\mathrm{MeV}^{-1}$. The analysis of the IA and the MEC contributions are performed separately in the same fashion that has been implemented for the m.m.'s.

The predictions for the $A=6,7$ nuclei as well as those for the $A=8,\left(1^{+} \rightarrow 2^{+}\right)$transitions are in very good agreement with the experimental data. In all these cases the MEC corrections are needed to bring the theory in agreement with the experimental data. Results for the $\left(3^{+} \rightarrow 2^{+}\right)$transitions in the $A=8$ systems underpredict the experimental data, however the latter have large experimental errors, and thus it is difficult to reach any robust conclusions as to the actual level of agreement between theory and experiment. The transition in ${ }^{9} \mathrm{Be}$ is known with good accuracy, but the predicted width is lower than the experimental data although the error bars almost touch. We also report a prediction for the $\left(\frac{1}{2}^{-} \rightarrow \frac{3}{2}^{-}\right) M 1$ transition in ${ }^{9} \mathrm{Li}$ which has not been measured yet. We did not calculate the ${ }^{9} \mathrm{C}$ transition to its unbound $\frac{1}{2}^{-}$state.

The magnetic transition densities in IA as obtained from the VMC starting wave functions are shown in Fig. 6. As before, the red upward-pointing triangles are the contribution from the proton spin term, the blue downward-pointing triangles are from the neutron spin, the green diamonds are from the proton orbital term, and the black circles are the total IA contribution. For the lithium isotopes, the transitions are predominantly from the proton spin term, i.e., these are almost pure proton spin-flip transitions. For ${ }^{7} \mathrm{Be}$ and ${ }^{8} \mathrm{~B}$, the neutron spin term is the most important, but with some contribution from the proton spin and orbital terms. The neutron spin-flip is also the biggest term in the ${ }^{9} \mathrm{Be}$ transition, but here the proton orbital piece is almost the same size.

Finally in Table $\mathrm{X}$, we show IA results for the electric quadrupole matrix elements and the associated transition widths. The latter in units of $\mathrm{MeV}$ is

$$
\Gamma_{E 2}=0.241\left(\frac{\Delta E}{\hbar c}\right)^{5} B(E 2),
$$

where $B(E 2)$ is the square of the reduced matrix element of the electric quadrupole operator given by

$$
\rho^{\mathrm{IA}}=\sum_{i} e_{N, i} r_{i}^{2} Y_{2}\left(\hat{\mathbf{r}}_{i}\right)
$$

where $Y_{L}$ is the spherical harmonic. The IA picture provides a decent description of the two experimental data points that are available, which might possibly be improved by the inclusion of two-body effects. This topic has not been addressed in this work although effort in this direction is underway.

The results discussed in this section are summarized in Fig. 7 for EM transitions whose widths are known experimentally. We observe that in most cases, the agreement with the experimental data is excellent, and that the MEC contributions are crucial for the $B(M 1)$ cases.

\section{CONCLUSIONS}

In this work we have reported GFMC results for EM moments and transitions of $A \leq 9$ nuclei. The calculations of m.m.'s and $M 1$ transitions account for the effect of two-body EM currents, for which we considered two models: i) the SNPA model described in Refs. 2, 10], and ii) the pionful $\chi$ EFT EM operator derived in [11 13]. The goals of this work were to continue the study initiated in Refs. [1, 2], by extending the calculations to systems with 
TABLE IX: Matrix elements in units of nuclear magnetons and widths of $M 1$ transitions in $A=6-9$ nuclei which account for the $\chi$ EFT current operator up to N3LO. IA and MEC contributions are shown.

\begin{tabular}{|c|c|c|c|c|c|}
\hline$\left(J_{i}^{\pi} \rightarrow J_{f}^{\pi}\right)$ & $M 1$ and $\Gamma$ & IA & MEC & Total & Expt. \\
\hline${ }^{6} \mathrm{Li}\left(0^{+} \rightarrow 1^{+}\right)$ & $\begin{array}{c}\mathrm{M} 1 \\
\Gamma(\mathrm{eV})\end{array}$ & $\begin{array}{l}3.63(1) \\
6.90(2)\end{array}$ & 0.38 & $\begin{array}{l}4.01(1) \\
8.41(3)\end{array}$ & $8.19(17)$ \\
\hline${ }^{7} \mathrm{Li}\left(\frac{1}{2}^{-} \rightarrow \frac{3}{2}^{-}\right)$ & $\begin{array}{c}\mathrm{M} 1 \\
\Gamma\left(10^{-3} \mathrm{eV}\right)\end{array}$ & $\begin{array}{l}2.66(1) \\
4.47(5)\end{array}$ & $0.47(1)$ & $\begin{array}{l}3.13(2) \\
6.19(8)\end{array}$ & $6.30(31)$ \\
\hline${ }^{7} \mathrm{Be}\left(\frac{1}{2}^{-} \rightarrow \frac{3}{2}^{-}\right)$ & $\begin{array}{c}\mathrm{M} 1 \\
\Gamma\left(10^{-3} \mathrm{eV}\right)\end{array}$ & $\begin{array}{l}2.31(2) \\
2.44(4)\end{array}$ & $0.41(1)$ & $\begin{array}{l}2.72(2) \\
3.39(6)\end{array}$ & $3.43(45)$ \\
\hline${ }^{8} \mathrm{Li}\left(1^{+} \rightarrow 2^{+}\right)$ & $\begin{array}{c}\mathrm{M} 1 \\
\Gamma\left(10^{-2} \mathrm{eV}\right)\end{array}$ & $\begin{array}{l}3.47(4) \\
4.4(1)\end{array}$ & $0.74(2)$ & $\begin{array}{l}4.21(5) \\
6.5(2)\end{array}$ & $5.5(1.8)$ \\
\hline${ }^{8} \mathrm{~B}\left(1^{+} \rightarrow 2^{+}\right)$ & $\begin{array}{c}\mathrm{M} 1 \\
\Gamma\left(10^{-2} \mathrm{eV}\right)\end{array}$ & $\begin{array}{l}3.17(5) \\
1.8(1)\end{array}$ & $0.67(2)$ & $\begin{array}{l}3.84(6) \\
2.6(1)\end{array}$ & $2.52(11)$ \\
\hline${ }^{8} \mathrm{Li}\left(3^{+} \rightarrow 2^{+}\right)$ & $\begin{array}{c}\mathrm{M} 1 \\
\Gamma\left(10^{-2} \mathrm{eV}\right)\end{array}$ & $\begin{array}{l}0.98(6) \\
1.8(2)\end{array}$ & $0.20(5)$ & $\begin{array}{l}1.17(8) \\
2.6(3)\end{array}$ & $7.0(3.0)$ \\
\hline${ }^{8} \mathrm{~B}\left(3^{+} \rightarrow 2^{+}\right)$ & $\begin{array}{c}\mathrm{M} 1 \\
\Gamma\left(10^{-2} \mathrm{eV}\right)\end{array}$ & $\begin{array}{l}1.31(6) \\
3.5(3)\end{array}$ & $0.23(5)$ & $\begin{array}{l}1.56(8) \\
4.9(5)\end{array}$ & $10(5)$ \\
\hline${ }^{9} \operatorname{Li}\left(\frac{1}{2}^{-} \rightarrow \frac{3}{2}^{-}\right)$ & $\begin{array}{c}\mathrm{M} 1 \\
\Gamma\left(10^{-1} \mathrm{eV}\right)\end{array}$ & $\begin{array}{l}2.28(3) \\
5.9(2)\end{array}$ & $0.36(4)$ & $\begin{array}{l}2.64(5) \\
7.9(3)\end{array}$ & n.a. \\
\hline${ }^{9} \mathrm{Be}\left(\frac{5}{2}^{-} \rightarrow \frac{3}{2}^{-}\right)$ & $\begin{array}{c}\mathrm{M} 1 \\
\Gamma\left(10^{-2} \mathrm{eV}\right)\end{array}$ & $\begin{array}{l}1.42(3) \\
5.6(3)\end{array}$ & $0.20(2)$ & $\begin{array}{l}1.62(4) \\
7.2(4)\end{array}$ & $8.9(1.0)$ \\
\hline
\end{tabular}

$A>7$, and to test the $\chi$ EFT two-body EM current operator within a hybrid context. Both models describe the long-range behavior of the two-body EM current in terms of OPE contributions. These pseudoscalar terms constitute the major contribution to the total MEC correction. The models also include, albeit in different formulations, the effects due to currents involving $\Delta$ isobar degrees of freedom. While the SNPA current does not involve free parameters, the $\chi$ EFT EM operator invokes a number of unknown LECs which have been fixed to reproduce EM observables in the $A=2,3$ nuclei. This additional freedom is probably responsible for the closer agreement with experiment given by the $\chi \mathrm{EFT}$ formulation compared to the SNPA model. In particular, both the isoscalar and isovector MEC corrections for the m.m.'s are closer to experimental data when calculated with the $\chi$ EFT EM currents. Nevertheless, we find that the two models are in good qualitative agreement and both support the necessity of adding MEC corrections to reach agreement with the experimental data.

In view of the study presented in Ref. [45] and ref- erences therein, we have paid special attention to the ${ }^{9} \mathrm{Li},{ }^{9} \mathrm{C}$ mirror nuclei. The experimental value for the isoscalar spin expectation value, $\left\langle\sigma_{z}\right\rangle=1.44$, has been considered 'anomalous' and various explanations, including a broken mirror symmetry, have been suggested. We find that calculations of $\left\langle\sigma_{z}\right\rangle$ obtained assuming mirror symmetry are close to the single-particle estimate of 1 , even if MEC contributions are included. When the mirror nuclei wave functions are constructed individually, including the appropriate Coulomb differences in both the starting VMC trial function and GFMC propagator, and thus breaking mirror symmetry, both the IA and MEC components of $\left\langle\sigma_{z}\right\rangle$ are increased in the right direction, giving a result that is consistent with the experimental value, and indicating that both broken symmetry and MEC contributions are required. However, the error associated with this last calculation of the $\left\langle\sigma_{z}\right\rangle$ value is too large to make a definitive statement at this stage.

Finally, we have studied a number of EM transitions induced by the $M 1$ and $E 2$ operators. After including the MEC contributions, the calculated $M 1$ transition widths 


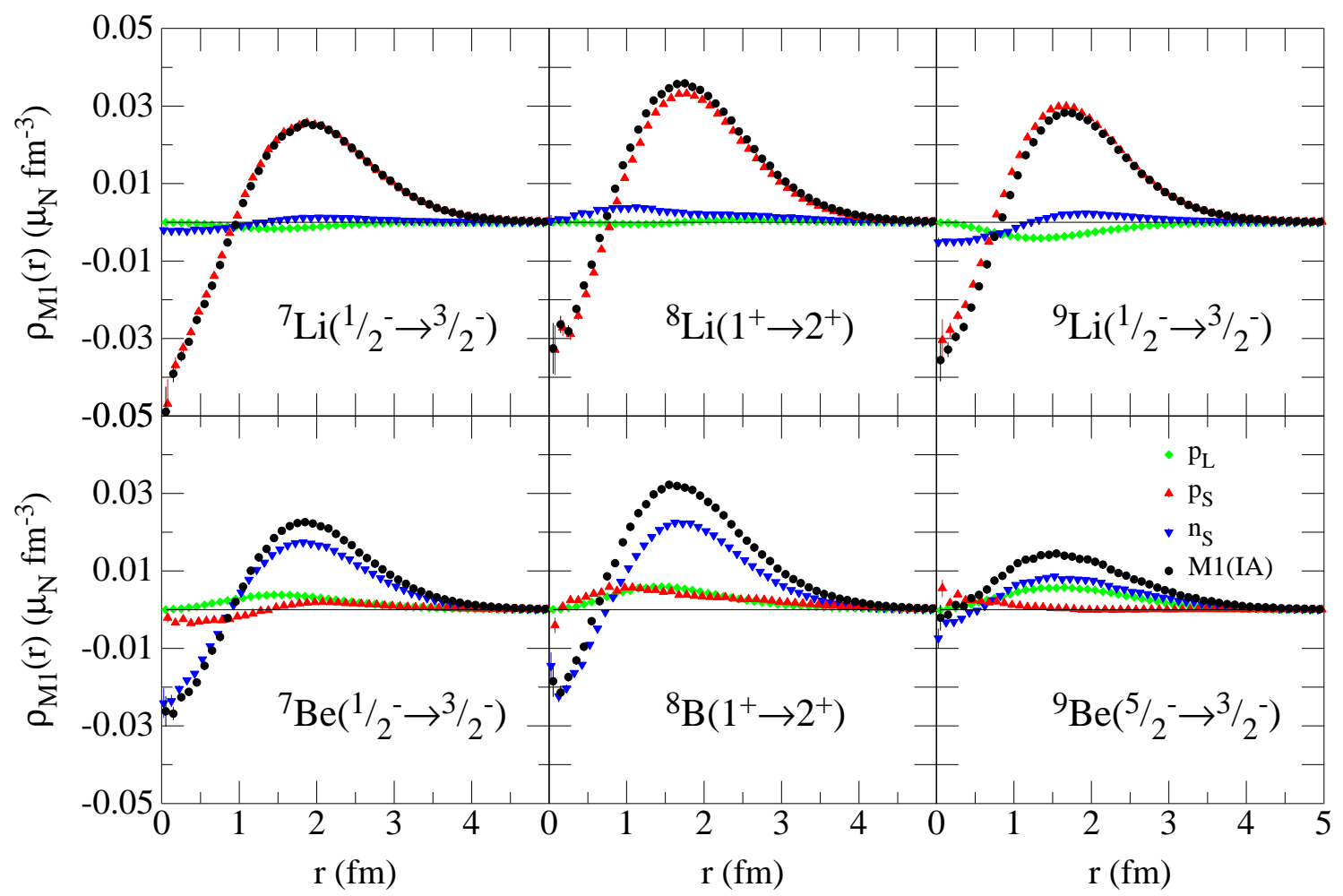

FIG. 6: (Color online) $M 1$ transition density in nuclear magnetons per $\mathrm{fm}^{3}$ for selected nuclei (see text for explanation).

are in excellent agreement with the experimental data for the $A=6,7$ nuclei and the $A=8\left(1^{+} \rightarrow 2^{+}\right)$cases, while theory underpredicts the data in the $A=8\left(3^{+} \rightarrow 2^{+}\right)$ and $A=9$ cases. However, the latter $A=8$ transitions have large experimental errors, so new precise precise measurements for these as well as for the as yet unmeasured ${ }^{9} \operatorname{Li}\left(\frac{3}{2}^{-} \rightarrow \frac{1}{2}^{-}\right)$transition would be most useful. For the $E 2$ transitions we provide only IA results. While two-body corrections to the IA charge operator have been derived in both SNPA [48, 49] and $\chi \mathrm{EFT}$ [50], nevertheless their leading contribution due to OPE is expected to be small, because the associated operator vanishes in the static limit. The present IA calculations appear to describe satisfactorily the $E 2$ transition widths for both the ${ }^{7} \mathrm{Li}\left(\frac{1}{2}^{-} \rightarrow \frac{3}{2}^{-}\right)$and ${ }^{9} \mathrm{Be}\left(\frac{5}{2}^{-} \rightarrow \frac{3}{2}^{-}\right)$cases.

\section{Acknowledgments}

R.S. would like to thank the T-2 group in the Theoretical Division at LANL, and especially J. Carlson and
S. Gandolfi, for the support and warm hospitality extended to him during a sabbatical visit in the Fall 2012, during which part of this work was completed. The many-body calculations were performed on the parallel computers of the Laboratory Computing Resource Center, Argonne National Laboratory, the computers of the Argonne Leadership Computing Facility (ALCF) via an INCITE grant, and of the National Energy Research Scientific Computing Center (NERSC) at Livermore. This work is supported by the U.S. Department of Energy, Office of Nuclear Physics, under contracts No. DE-AC02-06CH11357 (S.P., S.C.P., and R.B.W.) and No. DE-AC05-06OR23177 (R.S.) and under the NUCLEI SciDAC-3 grant.
[1] M. Pervin, S. C. Pieper, and R. B. Wiringa, Phys. Rev. C 76, 064319 (2007).

[2] L. E. Marcucci, M. Pervin, S. C. Pieper, R. Schiavilla, and R. B. Wiringa, Phys. Rev. C 78, 065501 (2008).
[3] R. B. Wiringa, V. G. J. Stoks, and R. Schiavilla, Phys. Rev. C 51, 38 (1995).

[4] S. C. Pieper, V. R. Pandharipande, R. B. Wiringa, and J. Carlson, Phys. Rev. C 64, 014001 (2001). 
TABLE X: Matrix elements in units of $e \mathrm{fm}^{2}$ and widths of $E 2$ transitions in $A=7-9$ nuclei. Only IA results are shown.

\begin{tabular}{|c|c|c|c|}
\hline$\left(J_{i}^{\pi} \rightarrow J_{f}^{\pi}\right)$ & $E 2$ and $\Gamma$ & IA & Expt. \\
\hline${ }^{7} \mathrm{Li}\left(\frac{1}{2}^{-} \rightarrow \frac{3}{2}^{-}\right)$ & $\begin{array}{c}\mathrm{E} 2 \\
\Gamma\left(10^{-7} \mathrm{eV}\right)\end{array}$ & $\begin{array}{r}5.59(16) \\
3.1(2)\end{array}$ & $3.30(21)$ \\
\hline${ }^{7} \mathrm{Be}\left(\frac{1}{2}^{-} \rightarrow \frac{3}{2}^{-}\right)$ & $\begin{array}{c}\mathrm{E} 2 \\
\Gamma\left(10^{-7} \mathrm{eV}\right)\end{array}$ & $\begin{array}{r}9.43(24) \\
5.2(3)\end{array}$ & n.a. \\
\hline${ }^{8} \mathrm{Li}\left(1^{+} \rightarrow 2^{+}\right)$ & $\begin{array}{c}\mathrm{E} 2 \\
\Gamma\left(10^{-6} \mathrm{eV}\right)\end{array}$ & $\begin{array}{r}2.04(8) \\
1.0(1)\end{array}$ & n.a. \\
\hline${ }^{8} \mathrm{~B}\left(1^{+} \rightarrow 2^{+}\right)$ & $\begin{array}{c}\mathrm{E} 2 \\
\Gamma\left(10^{-6} \mathrm{eV}\right)\end{array}$ & $\begin{array}{r}4.40(16) \\
1.4(1)\end{array}$ & n.a. \\
\hline${ }^{8} \mathrm{Li}\left(3^{+} \rightarrow 2^{+}\right)$ & $\begin{array}{c}\mathrm{E} 2 \\
\Gamma\left(10^{-4} \mathrm{eV}\right)\end{array}$ & $\begin{array}{r}6.09(10) \\
2.5(1)\end{array}$ & n.a. \\
\hline${ }^{8} \mathrm{~B}\left(3^{+} \rightarrow 2^{+}\right)$ & $\begin{array}{c}\mathrm{E} 2 \\
\Gamma\left(10^{-4} \mathrm{eV}\right)\end{array}$ & $\begin{array}{r}8.64(23) \\
5.8(3)\end{array}$ & n.a. \\
\hline${ }^{9} \operatorname{Li}\left(\frac{1}{2}^{-} \rightarrow \frac{3}{2}^{-}\right)$ & $\begin{array}{c}\mathrm{E} 2 \\
\Gamma\left(10^{-4} \mathrm{eV}\right)\end{array}$ & $\begin{array}{r}3.69(9) \\
7.7(4)\end{array}$ & n.a. \\
\hline${ }^{9} \mathrm{Be}\left(\frac{5}{2}^{-} \rightarrow \frac{3}{2}^{-}\right)$ & $\begin{array}{c}\mathrm{E} 2 \\
\Gamma\left(10^{-3} \mathrm{eV}\right)\end{array}$ & $\begin{array}{r}12.39(15) \\
1.7(1)\end{array}$ & $1.89(14)$ \\
\hline
\end{tabular}

[5] S. C. Pieper and R. B. Wiringa, Annu. Rev. Nucl. Part. Sci. 51, 53 (2001).

[6] S. C. Pieper, K. Varga, and R. B. Wiringa, Phys. Rev. C 66, 044310 (2002).

[7] S. C. Pieper, R. B. Wiringa, and J. Carlson, Phys. Rev. C 70, 054325 (2004).

[8] S. C. Pieper, Nucl. Phys. A751, 516c (2005).

[9] S. C. Pieper, AIP Conf. Proc. 1011, 143 (2008).

[10] L. E. Marcucci, M. Viviani, R. Schiavilla, A. Kievsky, and S. Rosati, Phys. Rev. C 72, 014001 (2005).

[11] S. Pastore, R. Schiavilla, and J.L. Goity, Phys. Rev. C 78, 064002 (2008).

[12] S. Pastore, L. Girlanda, R. Schiavilla, M. Viviani, and R. B. Wiringa, Phys. Rev. C 80, 034004 (2009).

[13] M. Piarulli, L. Girlanda, L. E. Marcucci, S. Pastore, R. Schiavilla, and M. Viviani, Phys. Rev. C 87, 014006 (2013).

[14] R. B. Wiringa, Phys. Rev. C 43, 1585 (1991).

[15] B. S. Pudliner, V. R. Pandharipande, J. Carlson, S. C. Pieper, and R. B. Wiringa, Phys. Rev. C 56, 1720 (1997).

[16] N. Metropolis, A. W. Rosenbluth, M. N. Rosenbluth, A. H. Teller, and E. Teller, J. Chem. Phys. 21, 1087 (1953).

[17] J. Carlson, Phys. Rev. C 36, 2026 (1987).

[18] J. Carlson, Phys. Rev. C 38, 1879 (1988).

[19] R. B. Wiringa, S. C. Pieper, J. Carlson, and V. R. Pandharipande, Phys. Rev. C 62, 014001 (2000).

[20] T.-S. Park, D.-P. Min, and M. Rho, Nucl. Phys. A596,

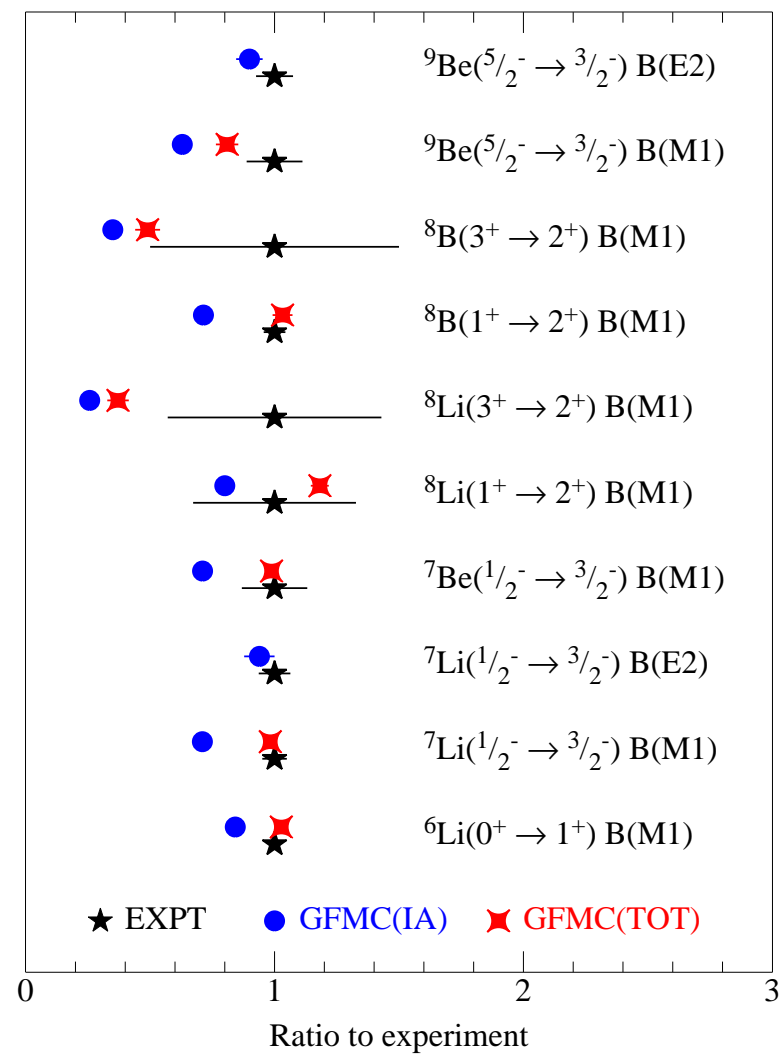

FIG. 7: (Color online) Ratio to the experimental $M 1$ and E2 transition widths in $A \leq 9$ nuclei. Black stars with error bars indicate the experimental values [35, 36], while blue dots (red diamonds) represent GFMC calculations which include the IA one-body EM current (total $\chi$ EFT current up to N3LO).

515 (1996).

[21] S. Kölling, E. Epelbaum, H. Krebs, U.-G. Meissner, Phys. Rev. C80, 045502 (2009).

[22] S. Kölling, E. Epelbaum, H. Krebs, and U.-G. Meissner, Phys. Rev. C 84, 054008 (2011).

[23] V.G.J. Stoks, R.G.E. Timmermans, J.J. de Swart, Phys. Rev. C 47, 512 (1993).

[24] R.A. Arndt, R.L. Workman, and M.M. Pavan, Phys. Rev. C 49, 2729 (1994).

[25] D.R. Entem and R. Machleidt, Phys. Rev. C 68, 041001 (2003); R. Machleidt and D.R. Entem, Phys. Rep. 503, 1 (2011).

[26] C. E. Carlson, Phys. Rev. D 34, 2704 (1986).

[27] A. Kievsky et al., Few-Body Syst. 22, 1 (1997).

[28] M. Viviani et al., Few-Body Syst. 39, 59 (2006).

[29] A. Kievsky et al., J. Phys. G: Nucl. Part. Phys. 35, 063101 (2008).

[30] B. S. Pudliner, V. R. Pandharipande, J. Carlson, and R. B. Wiringa, Phys. Rev. Lett. 74, 4396 (1995).

[31] R. Schiavilla, R.B. Wiringa, V.R. Pandharipande, and J. Carlson, Phys. Rev. C 45, 2628 (1992).

[32] G. P. Kamuntavičius, P. Navrátil, B. R. Barrett, G. Sapragonaite, and R. K. Kalinauskas, Phys. Rev. C 60, 044304 (1999).

[33] G. Audi, A. H. Wapstra, and C. Thibault Nucl. Phys. A 729, 337 (2003). 
[34] J. E. Purcell, J. H. Kelley, E. Kwan, C. G. Sheu, and H. R. Weller, Nucl. Phys. A 848, 1 (2010).

[35] D. R. Tilley, C. M. Cheves, J. L. Godwin, G. M. Hale, H. M. Hofmann, J. H. Kelley, C. G. Sheu, and H. R. Weller, Nucl. Phys. A 708, 3 (2002).

[36] D. R. Tilley, J. H. Kelley, J. L. Godwin,D. J. Millener, J. E. Purcell, C. G. Sheu, and H. R. Weller, Nucl. Phys. A 745, 155 (2004).

[37] D. Borremans, et al., Phys. Rev. C 72, 044309 (2005).

[38] P. J. Mohr, B. N. Taylor, and D. B. Newell, Rev. Mod. Phys. 84, 1527 (2012).

[39] A. Amroun et al., Nucl. Phys. A 579, 596 (1994).

[40] D. Shiner, R. Dixson, and V. Vedantham, Phys. Rev. Lett. 74, 3553 (1995).

[41] W. Nörtershäuser, T. Neff, R. Sánchez, and I. Sick, Phys. Rev. C 84, 024307 (2011).
[42] W. Nörtershäuser et al., Phys. Rev. Lett. 102, 062503 (2009).

[43] I. Brida, S. C. Pieper, and R. B. Wiringa, Phys. Rev. C 84, 024319 (2011).

[44] R. B. Wiringa, Phys. Rev. C 73, 034317 (2006).

[45] Y. Utsuno, Phys. Rev. C 70, 011303 (2004).

[46] K. Matsuta et al., Nulc. Phys. A 588, 153c; K. Matsuta et al., Hyperfine Interact. 97/98, 519 (1996).

[47] J. Carlson and R. Schiavilla, Rev. Mod. Phys. 70, 743 (1998)

[48] D.O. Riska, Phys. Rep. 181, 207 (1989).

[49] R. Schiavilla, V.R. Pandharipande, and D.O. Riska, Phys. Rev. C 41, 309 (1990).

[50] S. Pastore, L. Girlanda, R. Schiavilla, and M. Viviani, Phys. Rev. C 84, 024001 (2011). 